\title{
Large-scale circulation classification, weather regimes, and local climate over France, the Alps and Western Europe
}

\author{
Guy Plaut $^{1, *}$, Eric Simonnet ${ }^{2}$ \\ ${ }^{1}$ Institut Non Linéaire de Nice CNRS, Université de Nice - Sophia Antipolis, 1361 route des Lucioles, 06560 Valbonne, France \\ ${ }^{2}$ Laboratoire de Météorologie dynamique du CNRS, Ecole Polytechnique, 91128 Palaiseau, France
}

\begin{abstract}
By applying the dynamical cluster algorithm to large-scale circulation patterns at various tropospheric levels (Z700, Z500, and SLP [sea-level pressure]), we obtain the so-called weather regimes (WRs). WRs are the cluster central patterns; there is no subjectivity at all involved in the procedure. A red noise test allows one to select the best number of clusters at each level. A comparison is performed between the different level classifications, and highly significant correlations are found. While previous attempts to classify daily circulations in a fully objective way were concerned with mid-tropospheric levels and could not go further back than 50 yr, here we classify 120 yr of SLP patterns. We arrive at the important conclusion that the same 5 WRs are found for the 3 periods 1880-1918, 1919-1957 and 1958-1997. The linkage between these WRs and the local tangible weather is then investigated for both temperatures and precipitation. It is found that the instantaneous departure of local weather from average climate is highly correlated with the WRs, making this approach a challenging and coherent description of local climates. The atmosphere does not merely evolve around its mean state, but instead spends more time around a few peculiar (large-scale) states with specific consequences for local weather. As a result, the WRs may provide a reliable (and moreover fully objective) framework for building downscaling algorithms appropriate for local climate change studies.
\end{abstract}

KEY WORDS: Classification $\cdot$ Large-scale circulation $\cdot$ Weather regime $\cdot$ Local climate $\cdot$ European Alps · France

\section{INTRODUCTION}

Intriguingly, the name 'weather regime' (WR) has been tied to the most frequent, or the most persistent, mid-tropospheric large-scale circulation (LSC) patterns, not directly to tangible weather. The main purpose of this article is precisely to firmly state the linkage between the WRs and the actual local or mesoscale weather patterns over western Europe, especially over France and a few alpine sub-regions.

The concept of a WR was first introduced by synopticians in the early 1950 s; the idea being that, to a certain degree, the atmosphere evolves between a limited

*E-mail: plaut@inln.cnrs.fr number of states. Indeed, anyone living in western Europe during wintertime may observe long periods of mild wet and rainy westerlies. But sometimes the westerlies suddenly stop, and easterlies bring bitterly cold conditions. During some winters, easterlies almost never blow, but there are also winters with repeated easterly spells which may last for weeks, so that one could approximately say that there are 2 possible states for the atmosphere over the eastern Atlantic and western Europe: the westerly or zonal one and the easterly or blocked one. Indeed, during blocked periods, the mild westerlies (and the corresponding Atlantic lows) are stopped by long-lived (blocking) anticyclones over the North Sea or Scandinavia. The famous zonal-blocking transition over the Atlantic and 
Europe has been known for a long time; it was first interpreted as a manifestation of multiple-flow equilibria by Charney \& DeVore (1979).

The practical interest in the classification of (largescale) circulation maps into a few recurrent patterns lies in the observation that local tangible weather depends to a large extent on the large-scale flow. From the early 1950 s to the late 1970 s, the 'Grosswetterlagen' approach was developed in Germany (Gressel 1954a,b, 1959, Schepp 1979), whereas the Lamb weather types (Lamb 1972) were widely used by British synopticians. These classification schemes remain mostly subjective. Fully automatic and objective approaches only developed during the last 2 decades. Although one classifies circulation patterns, the name 'weather regime' became generic when referring to the patterns one obtains through objective classification algorithms. These algorithms may be gathered into approximately 3 categories:

(1) In the first one (Hansen \& Sutera 1986, Kimoto \& Ghil 1993a), WRs are looked for as those states that maximize the probability density function (PDF) in some low-dimensional spaces (typically the space spanned by the 2 to 5 leading PCs).

(2) The second approach is typified by the work of Vautard (1990) and aims at detecting the states for which the large-scale evolution is quasi-stationary in a statistical sense. Such states will naturally display a tendency to persist for a maximum time.

(3) The third approach relies on classification of large-scale patterns as clusters in a rather low-dimensional (but larger than in case 1) space (up to 10 dimensions). More confidence in this approach came with the development of long archives of data, currently encompassing 50 yr. Objective criteria were developed by Michelangeli et al. (1995) in order to select the best number of clusters and to check the significance of the classification.

One may wonder whether these different algorithms lead to similar WRs. Although no clear-cut conclusion can be drawn, it is noteworthy that Michelangeli et al. (1995), who used the dynamical cluster algorithm, a type 3 approach, obtained, over the Euro-Atlantic sector, 4 clusters which could be unambiguously put into a one-to-one correspondence with those found by Vautard (1990), who used a type 2 approach (statistical equilibration); the 2 sets of clusters displayed almost no qualitative differences. Kimoto \& Ghil (1993b) introduced a speed of evolution of the atmosphere. Their calculations show a clear tendency of the atmosphere to slow down when it evolves in the vicinity of their type 1 WR centers, which makes these centers also relevant candidates for a type 2 approach. In Section 7 , we will discuss the comparison of our type 3 algorithm WRs with types 1 and 2 .
Although our main interest in this work lies in the links between the WRs and the local climate (more precisely, the departure from local average climate), we first proceed to the classification of LSC patterns using 3 different large-scale fields. Prior works usually used only 1 such field, typically Z700 or Z500 heights, and could not compare the classes which emerge at different tropospheric levels. Here we look for WR patterns at 3 different levels: Z700, Z500, and SLP (sea-level pressure). We also use more homogeneous data sets, since geopotential heights have been reanalysed. Moreover, the $120 \mathrm{yr}$ of daily SLP patterns enable us to compare the WRs found for different historical periods.

In order to display the influence of a given WR on local climate, we will use the common procedure in which temperatures or precipitation are classified into quantiles. For instance, one expects that, with a zonal flow over western Europe, the warm tercile occurrence will dominate over northern France, or the last decile of precipitation will not occur over the Mediterranean flank of the Alps, whereas blocking easterlies will dry northern France, but favour intense precipitation over southern coastal regions exposed to Mediterranean easterlies, such as Catalonia or Roussillon. We will try to quantify such effects in Sections 5 and 6, in order to test the efficiency of the WR approach in the description of local weather rather than only in the description of local climate considered as some average weather. If the instantaneous departure of local weather from average climate may be reliably assigned to the specific WR present, this suggests that the WR approach may be an efficient one for building so-called downscaling algorithms. Downscaling infers local weather from LSC patterns in a more or less statistical way. It may be an appropriate tool for inferring local climate change from general circulation model (GCM) simulations, but it could also offer an alternative to purely deterministic forecasts when a firm link exists between LSC and local weather.

The different data sets used for classification as well as for local climate studies are described in Section 2, together with our methodology. Then we proceed to LSC classification into WRs in Section 3. Section 4 is devoted to a comparison of weather regimes at different levels, and for different historical periods in the case of SLP. The remainder of our work is devoted to investigations of the links between the previously obtained WRs and local climates over France, the Alps, and western Europe, in terms of temperature (Section 5) and precipitation (Section 6). Important conclusions about the reliability of the WR approach to describe local climate as well as to build downscaling algorithms for local climate change studies are given in Section 7. 


\section{DATA AND METHODOLOGY}

Although we are aware that the name 'circulation regimes' could be more appropriate, we keep to common practice and call the cluster central patterns we obtain when classifying all the LSC patterns of a data set in an objective way, without any a priori knowledge about the classes (including their number), 'weather regimes'. Our approach is type 3 (see Section 1), so we identify the most recurrent LSC patterns. As in Michelangeli et al. (1995), we use the dynamical cluster algorithm as implemented in our package ANAXV, which allows a fully objective procedure without any a priori hypothesis about the patterns to be found, or their number. This objectivity stands in contrast to the subjectivity of many older classification schemes (Gressel 1959, Lamb 1972, Schepp 1979), even if they were automated in their last step. But let us first describe the different large-scale fields we classify, allowing for a comparison of the WRs found at different levels, or for different periods.

2.1. LSC data sets. The daily geopotential heights $Z 500$ and $Z 700$ were obtained from the NCEP/NCAR reanalysis project and extend from 1958 until 1998 (Kalnay et al. 1996). They have been spatially extracted at the Climatic Research Unit, Norwich (UK). To make the comparison with the results of Michelangeli et al. (1995) easier, we classify winter month (November to March) daily LSCs, and use the same window of $100^{\circ}$ longitude and $40^{\circ}$ latitude, centered at $50^{\circ} \mathrm{N}, 10^{\circ} \mathrm{W}$, and covering most of the North Atlantic and Europe. With a resolution of $\left(2.5^{\circ} \times 2.5^{\circ}\right)$, it includes 640 gridpoints.

The SLP data were collected at the Climatic Research Unit; the grid resolution is $5^{\circ} \times 5^{\circ}$. They cover a much longer period (1880-1997), which permits very interesting comparisons between different periods. There are some known problems with these data, especially over very high terrain or the Arctic (Jones 1987). However, with the same window of $100^{\circ}$ longitude and $40^{\circ}$ latitude as above, these problems are quite irrelevant for our purpose.

2.2. Temperatures and precipitation data. In order to emphasize the links between the WRs and the actual weather, we use 4 temperature or precipitation data sets. These sets are of very different nature: the first 2 are made up of daily station archives; the third consists of daily gridded data built up from dense station network observations; and the fourth consists of gridded reanalysis data. More explicitly, we use:

- Mean daily temperatures recorded at 30 MétéoFrance stations from 1949 to 1996 . The locations are shown in Fig. 13.

- The same French stations' daily precipitation data.

- NCEP mean 2 m daily air temperatures (T2m) on a dense Gaussian grid, restricted to western Europe, extending from $30^{\circ}$ to $70^{\circ} \mathrm{N}$, and $10^{\circ} \mathrm{W}$ to $30^{\circ} \mathrm{E}$, and including 460 gridpoints. These data cover the period 1958-1997 and are reanalyzed data processed with the NCEP/NCAR model in the same way as the LSC geopotential heights.

- The Alpine Precipitation Climatology (hereafter APC) from the Swiss Federal Institute of Technology, Zürich (Frei \& Schär 1998), which covers the European Alps area and the adjacent foreland. It consists of a compilation of 6678 station records projected onto a $25 \mathrm{~km}$ grid for each day of the period 1971-1995. Each gridpoint corresponds on average to 6 stations. Even the gridded data offer very attractive possibilities for investigations of mesoscale climates, together with their links with the LSC.

2.3. Methodology. In order to classify LSCs, we must first reduce the dimension of the data set, since classification algorithms can only operate in low-dimensional spaces. This is not a problem, since we classify large-scale patterns: WRs are always looked for in the low-dimensional subspace spanned by the low-frequency/large-scale leading empirical orthogonal functions (EOFs) (Vautard 1990, Kimoto \& Ghil 1993b, Michelangeli et al. 1995). We thus perform a principal component analysis (PCA, based on the covariance matrix, including cosines of latitudes), and keep only a small number of EOFs, typically 10 . The precise number of EOFs kept is irrelevant, and almost indistinguishable patterns are obtained by keeping 6 to 10 EOFs or more: the WRs are essentially weakly unstable fixed points which lie in the low-frequency (actually lower than synoptic) subspace of the EOF space. Higher-order EOFs corresponding to higher-frequency/smaller scales simply do not contribute to the WR patterns.

We thus proceed to the classification of LSC patterns within this 10-dimensional PC space. Let us first assume that the number of clusters, $k$, is determined. Then the goal of the dynamical cluster algorithm is to find a partition of the whole data set into $k$ clusters that minimizes some criterion, usually the sum of variances within clusters. However, other criteria may be used as well (Robertson \& Ghil 1999), such as the sum of the the correlation distances $\left(d_{\mathrm{c}}\right)$ :

$$
d_{\mathrm{c}}=1-\mathrm{apcc}
$$

where apcc is the anomaly pattern correlation coefficient (actually a cosine in the PC space) and $d_{\mathrm{c}}$ ranges from 0 for perfectly correlated patterns up to 2 for inversely correlated ones. In Section 3.2, we briefly discuss the differences between the classifications obtained with the 2 kinds of criteria. The correlation distance $d_{c}$ is also used in Section 5.4 to impose more stringent class membership criteria. 
When achieving a particular classification, $k$, the number of clusters, is an a priori fixed parameter. However, we feel that the optimal value of $k$ should not be a subjective parameter, but should be determined by the data themselves, following an objective rule. Here, we adopt the rule proposed by Michelangeli et al. (1995). Starting from random seeds, the dynamical cluster algorithm finds partitions of the data into clusters. Let us call $P$ and $Q$ two such partitions of the same data set into $k$ clusters, starting from different random seeds. If $k$ has its optimal value, one would expect $P$ and $Q$ to look very similar, whatever the random seeds. In order to quantify this requirement, one defines a classifiability index $C^{*}$ which has to be as high as possible. In practice, one uses a red noise test and requires that the 'classifiability' of the true data be better than that of an ensemble of artificial data sets generated through a first-order Markov process having the same lag 0 and lag 1 covariance matrices as the true atmospheric data.

The red noise test operates in the following way: for a fixed value of $k$, and given any 2 partitions $P$ and $Q$, we compute $C(P, Q)$, a measure of the similarity between these 2 partitions, which is equal to 1 if the cluster central patterns of $P$ and $Q$ are identical and to -1 in the worst cases. This is done by first computing the apcc between any cluster centroid belonging to $P$ and any belonging to $Q$. For each cluster belonging to $P$, one selects its best $Q$ partner (that with the highest apcc), and $c(P, Q)$ is taken as the minimum of these highest apcc. This means that $c(P, Q)$ measures the correlation of the worse matching pair when matching $P$ and $Q$ into corresponding patterns. Fifty classifications of the true data set are generated, and the average $C^{*}$ of all the $C(P, Q)$ gives its classifiability index into $k$ clusters. In order to set significance limits for $C^{*}$, we use the first-order Markov process of Michelangeli et al. (1995): 100 samples of the same length as the true data set are generated, which give 100 values of $c^{*}$, which are ranked to find the $90 \%$ confidence limits. Then we compare the index $c^{*}$ of the true data set with these bounds: values of the atmospheric index above the upper bound indicate a classifiability significantly higher than for the red noise model. Most often, a best value of $k$ clearly appears.

\section{THE WEATHER REGIMES}

\section{1. $Z 700$ and $Z 500$ WRs}

We first apply the above procedure, which sets the confidence level bounds. A total of 6140 winter $Z 700$ maps were to be classified. Fig. 1 b displays $c^{*}$, the classifiability index, versus $k$, the number of classes,

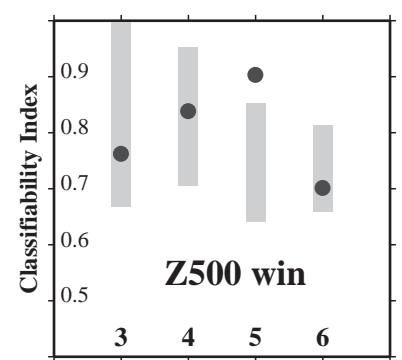

a)

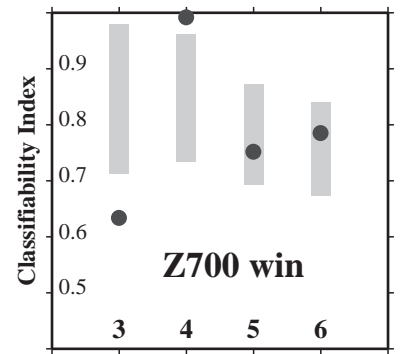

b) Number of Classes

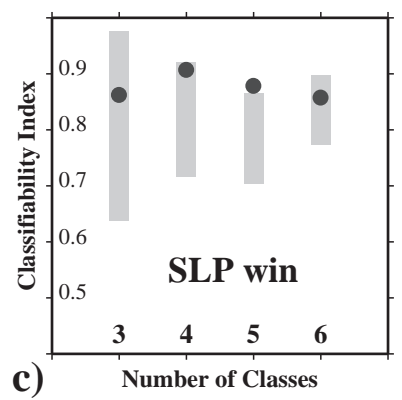

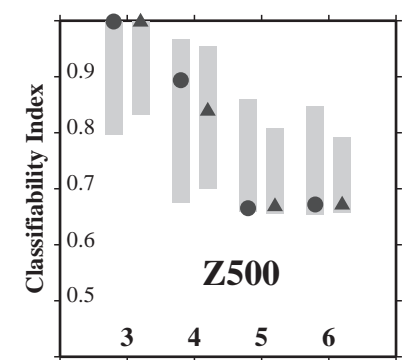

d)

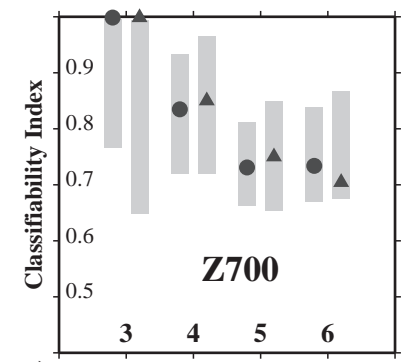

e)

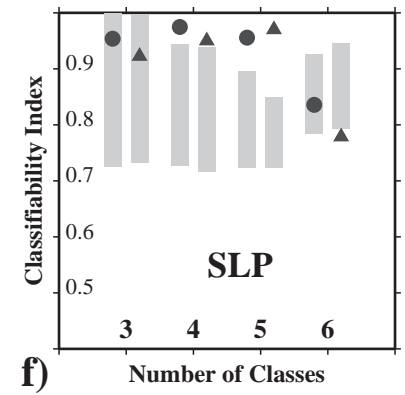

Fig. 1. $(\mathrm{a}, \mathrm{b}, \mathrm{c})$ Red noise significance checks for classification of extended winter (win) daily large-scale circulations (LSCs) into weather regimes (WRs). SLP: sea-level pressure; black circles: classifiability index, $\mathrm{C}^{*}$; grey bars: 10 to $90 \%$ confidence levels. (d,e,f) Red noise significance checks for classification over the whole year. Black circles and grey bars: classification using Euclidean metrics as in $(a, b, c)$; triangles: classification using the correlation distance $d_{\mathrm{c}}$ (see Section 3 for details)

together with the 10 to $90 \%$ bounds. Only the choice $k$ $=4$ leads to significant classes from the point of view of the red noise test. The classifiability index $c^{*}$ is then so high (0.99) that the 4 cluster central patterns of Fig. 2 do not display any dependence on the initial random seeds. These patterns are obviously the same as those found by Michelangeli et al. (1995). They display only minor differences from the category 2 WRs of Vautard (1990), which were looked for as quasi-stationary points in the 10-dimensional space spanned by the 10 leading PCs. They actually persist for about $4 \mathrm{~d}$ on average. Following Vautard (1990) and what is common practice, we name these patterns in the following way: AR (Atlantic Ridge), inducing north-westerlies 


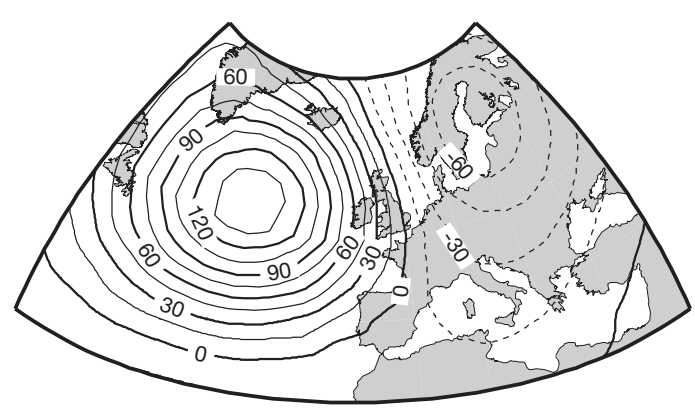

AR

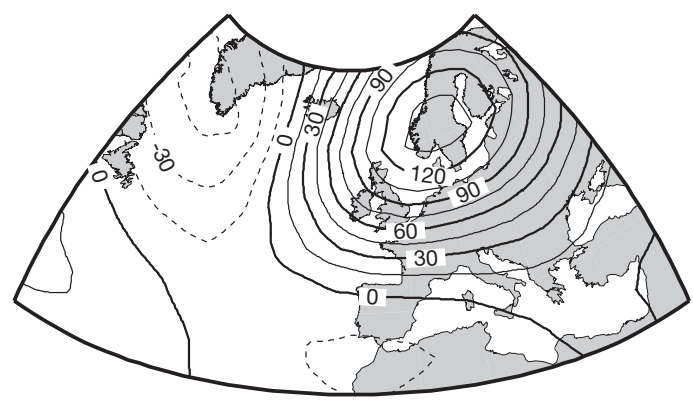

$(25 \%)$

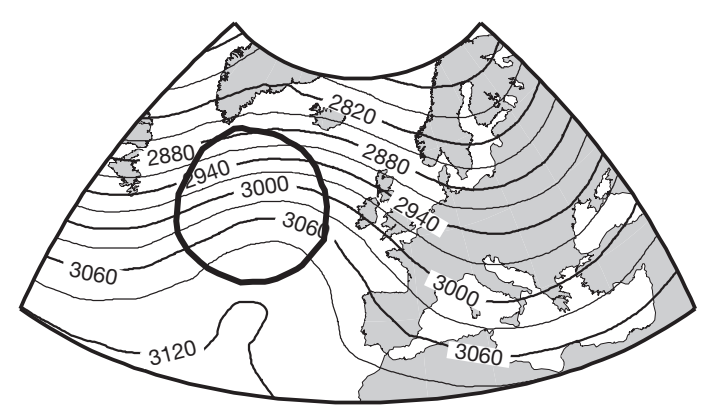

$\mathrm{BL}$

(27\%)
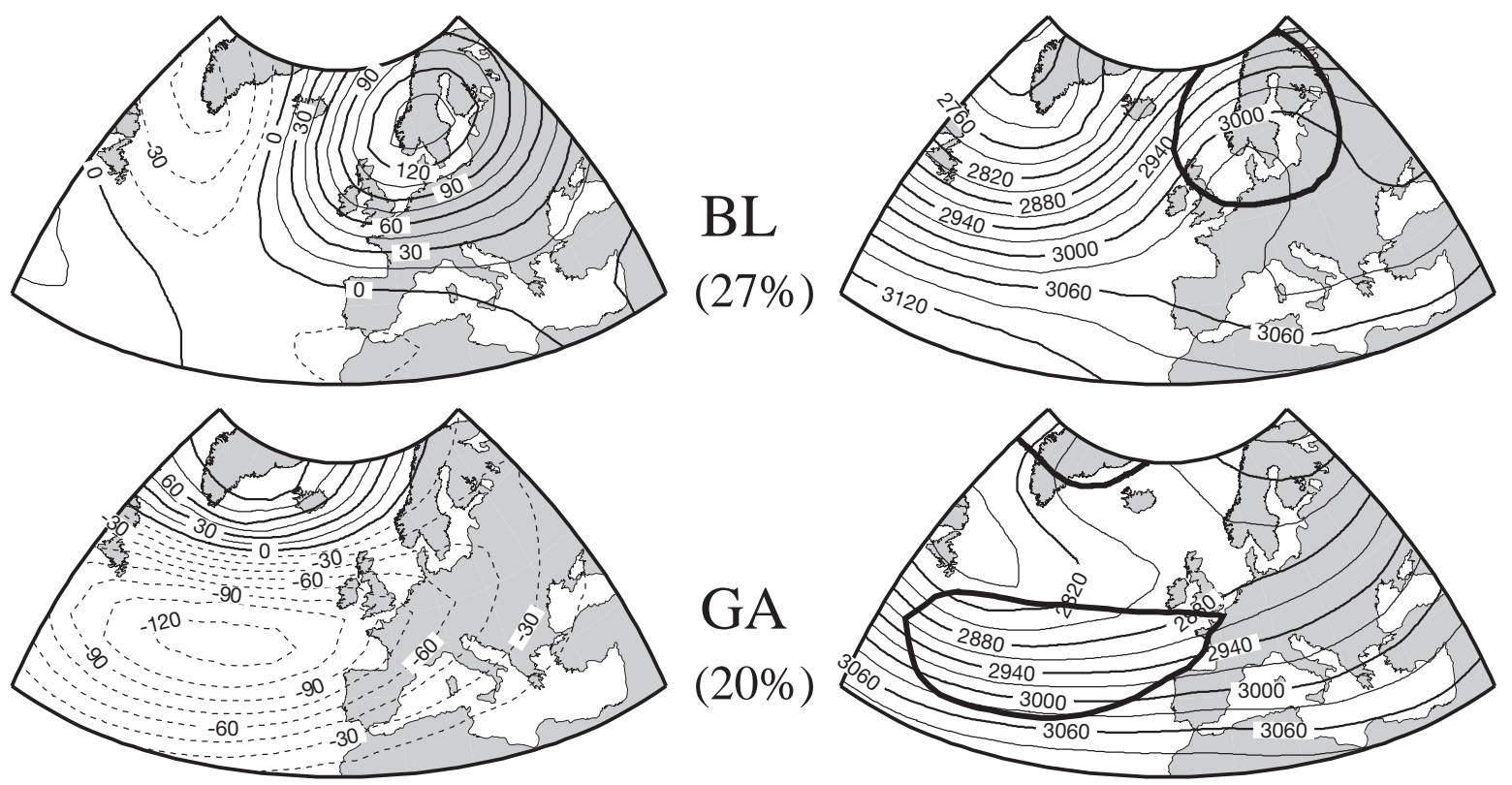

GA

$(20 \%)$
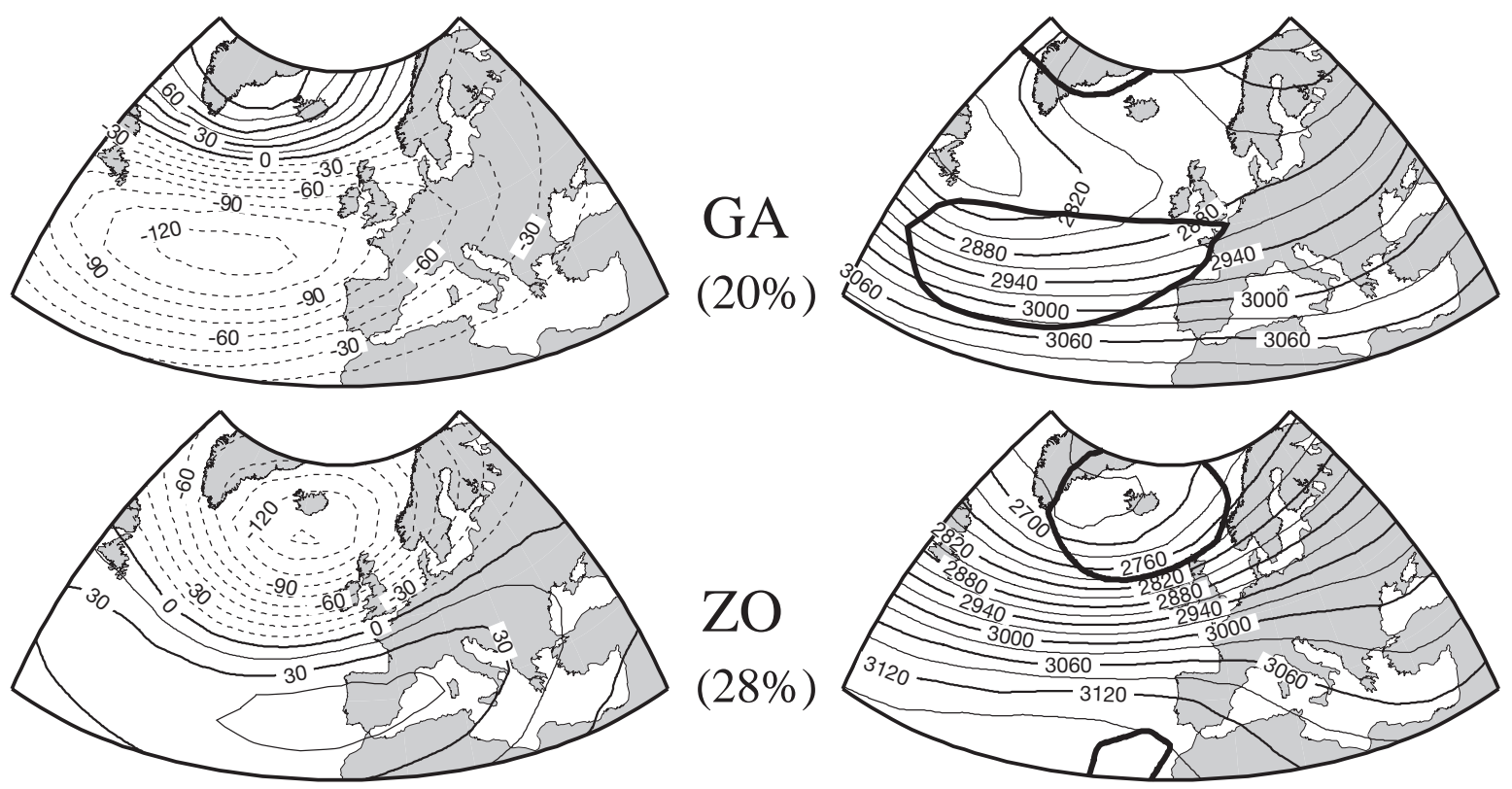

ANOMALIES

$\mathrm{ZO}$

$(28 \%)$

FULL FIELD

Fig. 2. The 4 Z700 WRs for winters (November to March) between 1958 and 1998. Left-hand column: cluster center anomalies Right-hand column: full Z700 fields of cluster centers (gpm). Parentheses: percentages of days in each class. AR: Atlantic Ridge; BL: Blocking; GA: Greenland Anticyclone; ZO: Zonal

over western and central Europe; BL (Blocking), with a maximum positive anomaly over Scandinavia; GA (Greenland Anticyclone), with a positive anomaly over Greenland and a negative one over the mid-latitude North Atlantic; and ZO (Zonal), with an enhanced zonal flow over the North Atlantic. The percentages of days within each class are also displayed in Fig. 2; the thick lines in the right-hand column indicate the areas where the cluster central pattern anomaly exceeds the intra-cluster variance.

The $Z 500$ maps to be classified span the same period as the $Z 700$. With $k=4$ clusters, the patterns correspond exactly to the $Z 700$ ones: correlations between the anomalies of the matching pairs of $Z 700$ and $Z 500$ 
cluster central patterns are all larger than $90 \%$ : the apcc defined in Section 2.3 is equal to 0.91 . However, the red noise test indicates that $k=5$ is preferred (Fig. 1a). The fifth selected regime displays a strong positive anomaly close to Newfoundland, together with a less important one over eastern Europe, and may be tentatively referred to as GT (Greenwich Trough). This regime does not persist when all-year LSCs are classified, and therefore appears less robust than others. The anomaly patterns for the 5 WRs are displayed in Fig. 3, together with the corresponding full Z500 patterns.

\subsection{SLP WRs}

Although the daily SLP data cover $120 \mathrm{yr}$, we first only classify winters after 1957 in order to make comparisons with $Z 700$ or $Z 500$. The choice $k=5$ classes is preferred (Fig. 1C). It may be seen in Fig. 4 that 4 regimes have anomaly patterns quite similar to the 4 $Z 700$ ones. The most outstanding difference relies on the fact that the SLP regime we call BL corresponds on average to an anticyclonic cell with its center over Eastern Europe near the Ukraine. An inspection of its central pattern in the right-hand column of Fig. 4 suggests that this cluster could be a mix of the well-known tropospheric BL and the famous Siberian SLP anticyclone, the western fringe of which overlaps the most eastern areas of our Euro-Atlantic domain.

To our knowledge, the SLP patterns have not been classified into WRs prior to this work, although many subjective classifications exist. We are thus faced with the problem of referring to each of them by some name. We are aware that the name 'European Anticyclone' (EA) could also have been tied to the WR we call BL. In the same way, there is some arbitrariness in choosing the name West Blocking (WBL) for the fifth regime, which could have been called simply Blocking. WBL actually favours much colder conditions over Europe, with an anticyclonic cell centered over Scotland on average.

Before turning to a comparison of WRs at different levels, let us briefly mention that we also performed daily LSC classification into WRs using all months of the year. In this case, each grid point seasonal cycle was removed prior to the PCA. Very similar patterns (not shown) were found. The red noise significance tests (leading to the best choice for $k$ ) are summarized in the right-hand column of Fig. 1. Three points are worth emphasizing: (1) Using an angular distance or a Euclidean one as above leads to identical conclusions for $k$, and almost undistinguishable classifications. (2) For $Z 700$ and $Z 500, k=3$ clusters are preferred; the patterns are identical at both levels, 2 of them being the already known as BL and ZO and a third one which may be called AR-GA. (3) Five clusters are always preferred for SLP; they are remarkably similar to the winter classes.

\section{COMPARISON AT DIFFERENT LEVELS AND FOR DIFFERENT PERIODS}

\subsection{Comparison between different level WRs}

The comparison between WRs at different levels may be tackled from 2 complementary points of view: one can compare the (anomaly) patterns, but one can also look at the contingency tables of simultaneous occurrences of the WRs found at 2 distinct levels. Insistence is put on space with the first approach, and on time with the second. From the spatial point of view, the similarities between 4 out of the 5 WRs of Fig. 3 and the 4 WRs of Fig. 2 are obvious, and this is confirmed by apcc calculations: the values of apcc for the matching pairs are all larger than $90 \%$.

However, especially for comparisons between SLP WRs and mid-tropospheric ones, the second point of view may be more appropriate: to a cold sea-level anticyclone such as the Siberian one, there does not necessarily correspond an upper-level positive anomaly. We adopt the latter point of view and compute contingency tables of WR occurrences at different levels (Tables 1 to 3 ). The 1 and 99\% significance thresholds were established by randomly shuffling the days 500 times. Numbers in italics are below the $1 \%$ confidence level threshold and refer to a smaller number of coincidences than random; bold numbers are above the $99 \%$ level. The significance thresholds are printed in small characters in parentheses below the actual number of coincidences: the high significance of all the values in Tables 1 to 3 clearly appears in this way. But let us comment on the results in more detail:

- Z700-Z500 (Table 1)-The 4 regimes AR, BL, GA and $Z O$ for $Z 700$ and $Z 500$ are in obvious one-to-one correspondence. This was suggested by visual inspection of Figs $2 \& 3$. The GT WR seems to correspond to a mix of the $Z 700 \mathrm{AR}$ and BL.

- Z700-SLP (Table 2) - Similar conclusions hold when we compare the $Z 700$ and SLP data; WBL appears to be a mix of certain $Z 700 \mathrm{AR}$ and BL members. It appears that the SLP BL coincides, in $20 \%$ of cases, with the $Z 700$ zonal regime. Indeed, an inspection of Fig. 4 reveals that over most of the North Atlantic, this regime corresponds to a zonal flow which may well coincide with an extension of the Siberian anticyclone over continental Europe and/or with longlived low-layer temperature inversions over this area as in January 1997. 


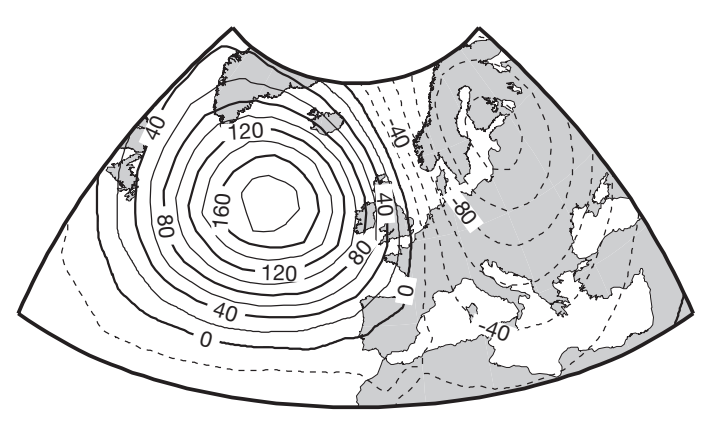

AR

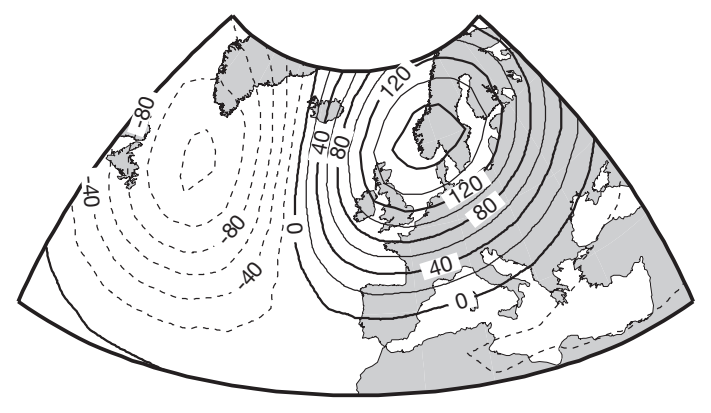

$(20 \%)$

$\mathrm{BL}$

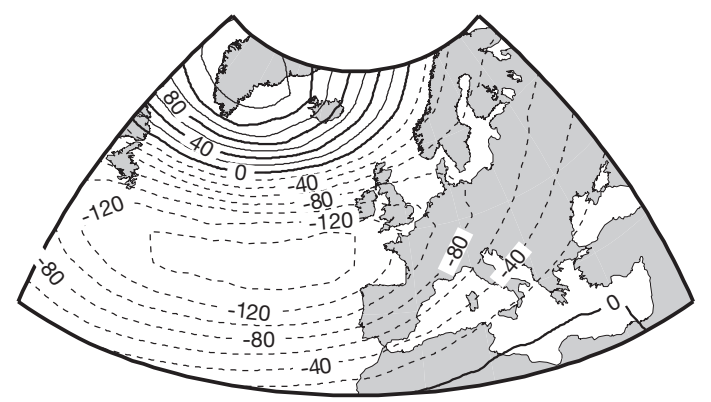

$(22 \%)$
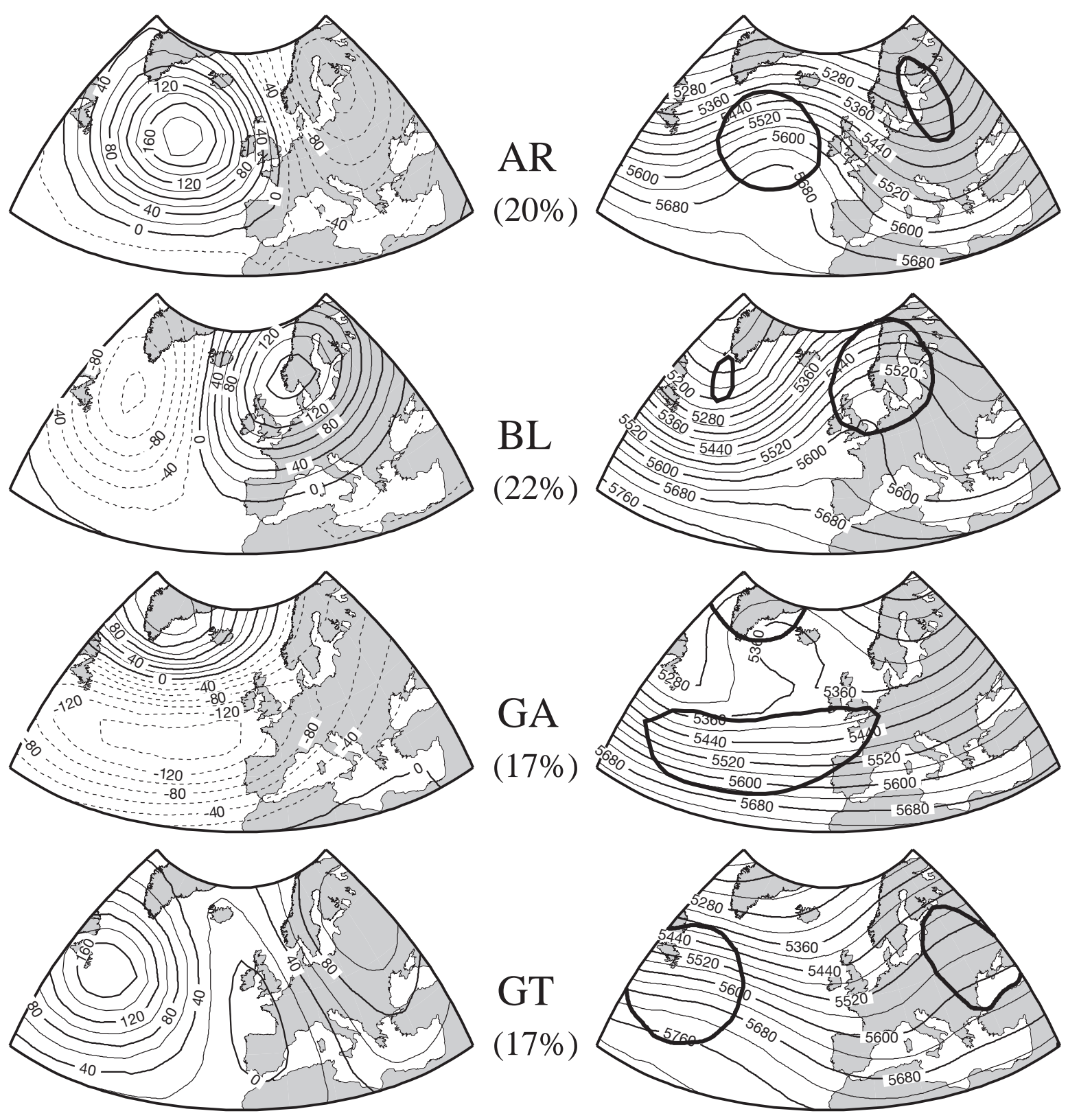

GA

$(17 \%)$

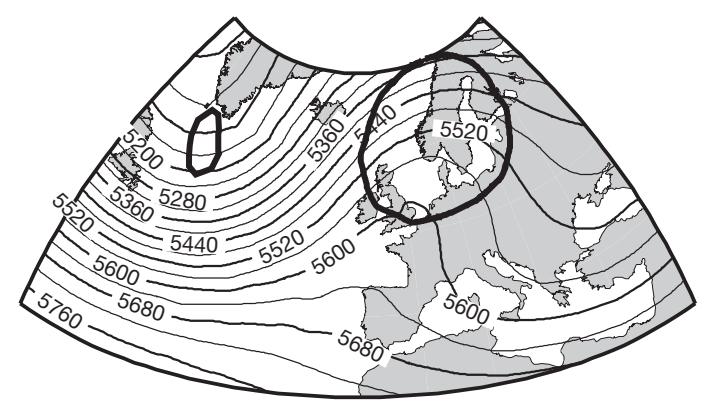

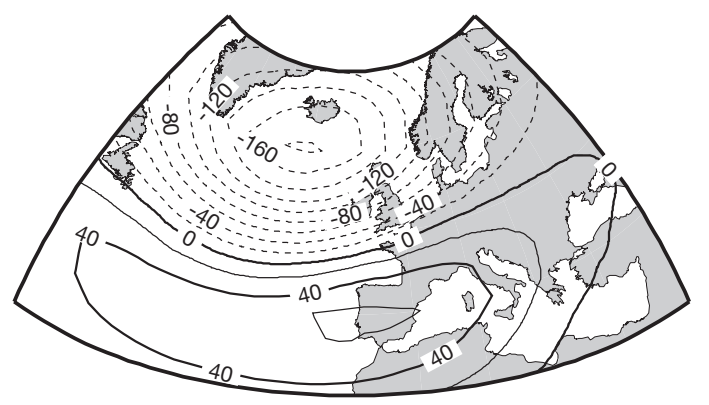

ANOMALIES
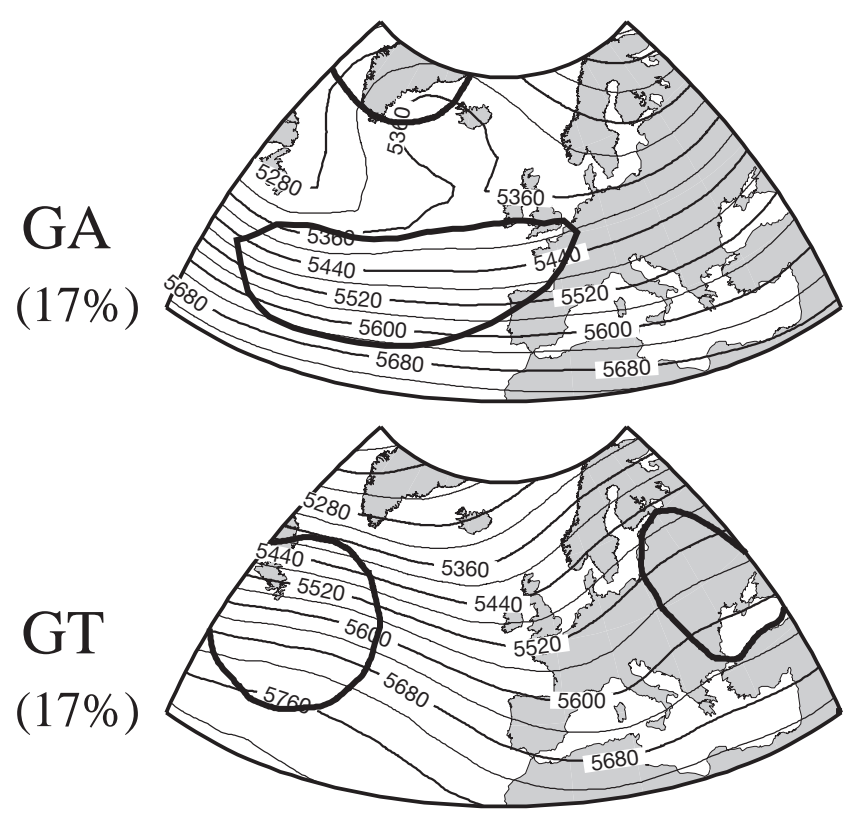

$\mathrm{ZO}$

(24\%)

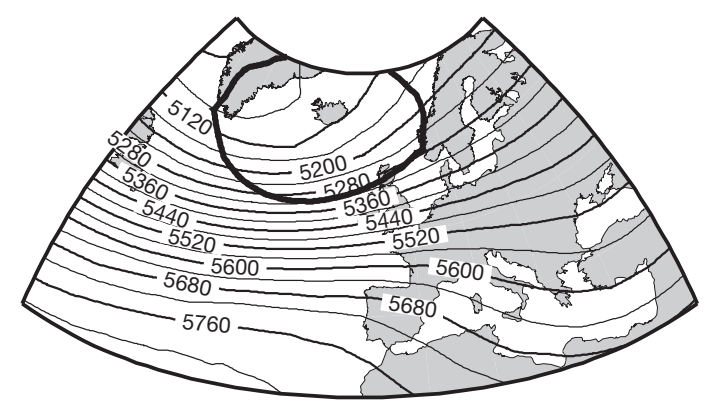

\section{FULL FIELD}

Fig. 3. The 5 Z500 WRs for winters (November to March) between 1958 and 1998. Left-hand column: cluster center anomalies. Right-hand column: full Z500 fields of cluster centers (gpm). Parentheses: percentages of days in each class. GT: Greenwich Trough; other labels as in Fig. 2 


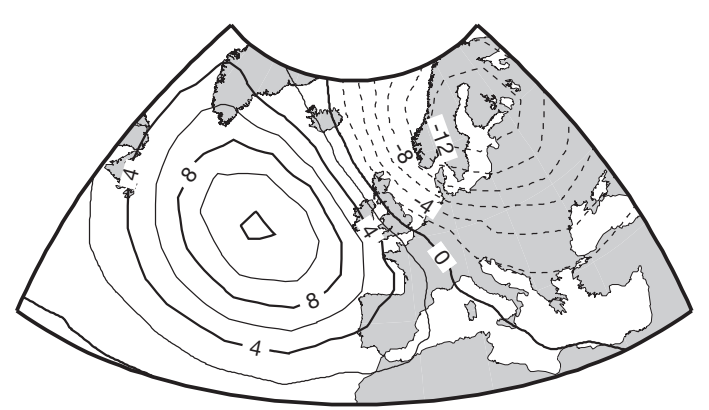

AR

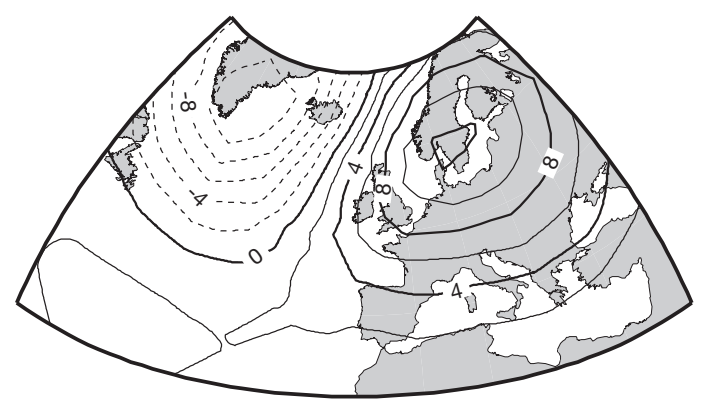

$(21 \%)$
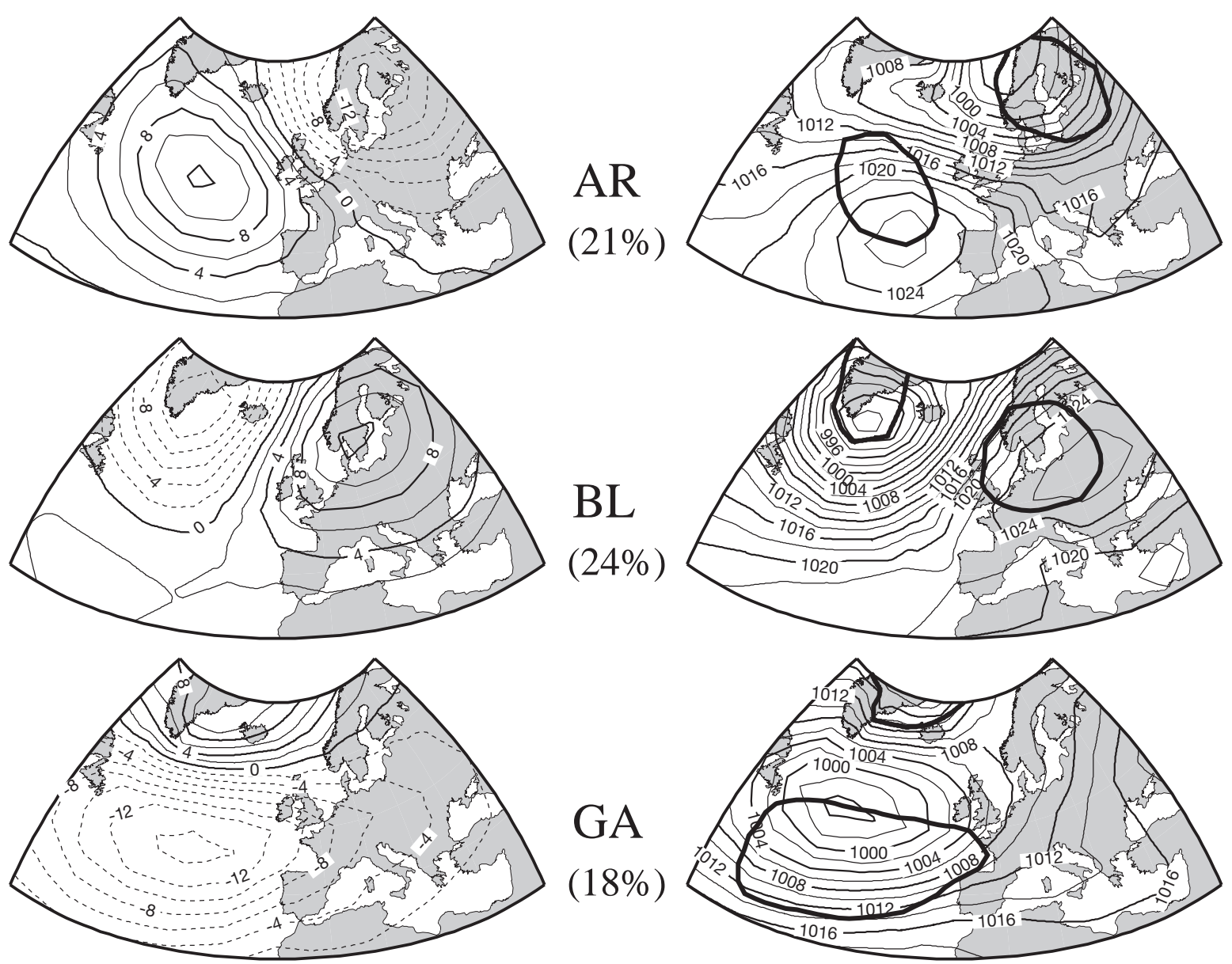

$\mathrm{BL}$

$(24 \%)$

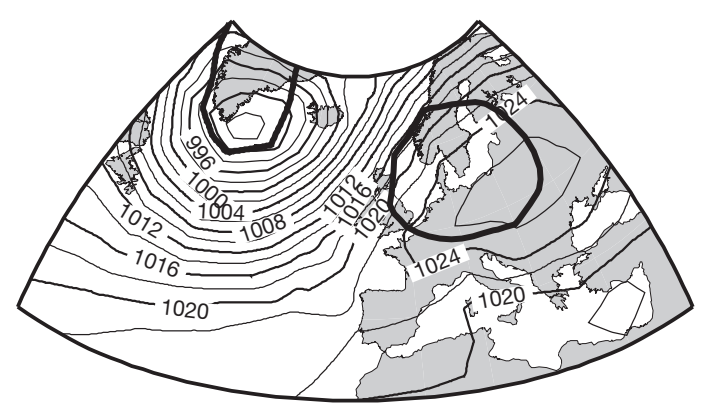

GA

$(18 \%)$
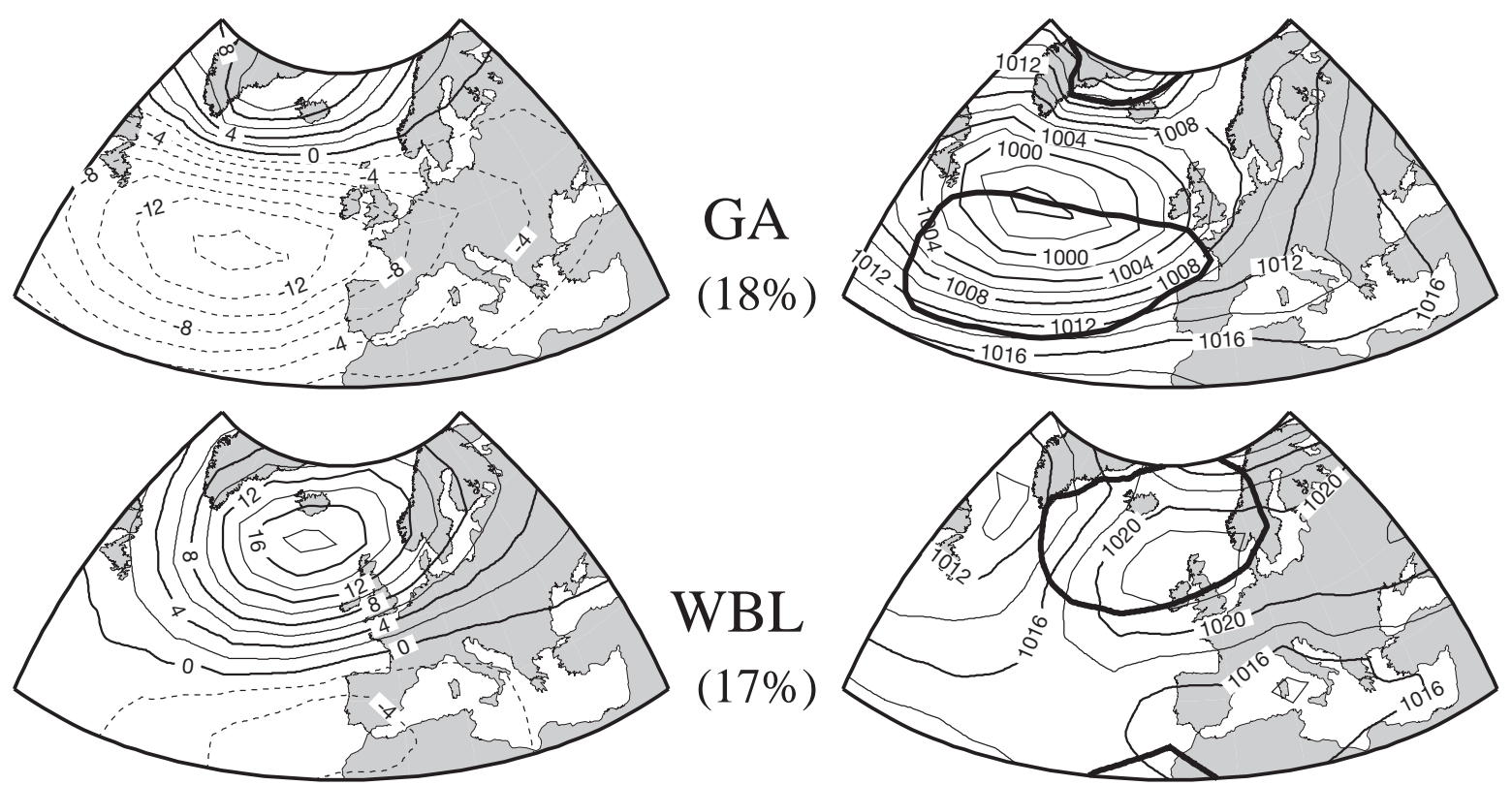

WBL

$(17 \%)$

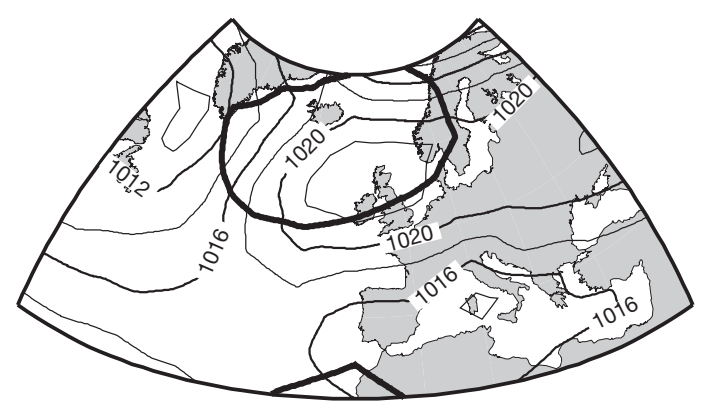

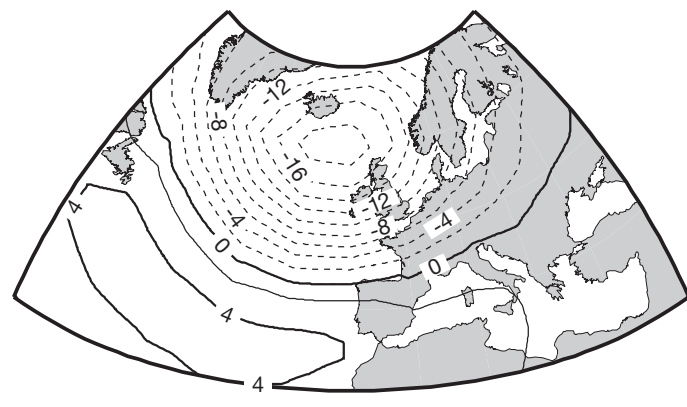

ANOMALIES

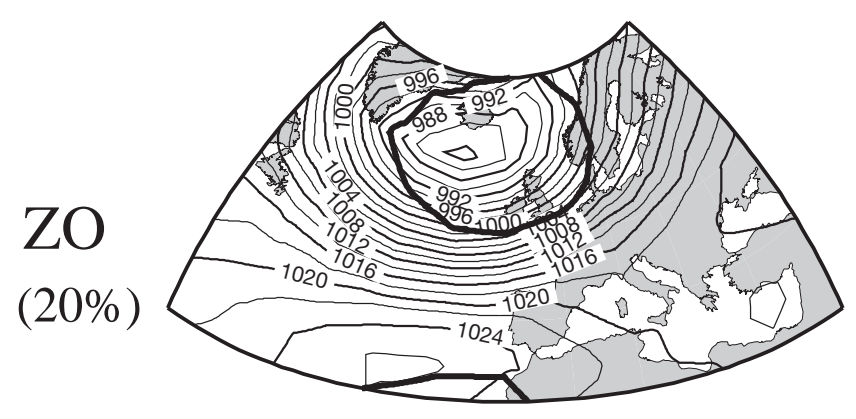

FULL FIELD

Fig. 4. The 5 SLP WRs for winters (November to March) between 1958 and 1998. Left-hand column: cluster center anomalies. Right-hand column: full SLP fields of cluster centers (hPa). Parentheses: percentages of days in each class. WBL: West Blocking; other labels as in Fig. 2 
Table 1. Contingency table of simultaneous occurrences of $Z 700$ (rows) and $Z 500$ (columns) WRs. To assess statistical confidence limits, the days were randomly shuffled 500 times. Values inside brackets span the 1 to $99 \%$ confidence level interval. Bold (italic) values correspond to statistically significant (at the $1 \%$ level) large (small) numbers of coincidences. AR: Atlantic Ridge; BL: Blocking; GA: Greenland Anticyclone; GT: Greenwich Trough; 20: Zonal

\begin{tabular}{|c|c|c|c|c|c|}
\hline$Z 700 / Z 500$ & $\mathrm{AR}$ & BL & GA & GT & $\mathrm{ZO}$ \\
\hline AR & $\begin{array}{c}1053 \\
(277-334)\end{array}$ & $\begin{array}{c}22 \\
(313-381)\end{array}$ & $\begin{array}{c}46 \\
(236-295)\end{array}$ & $\begin{array}{c}\mathbf{3 8 7} \\
(236-292)\end{array}$ & $\begin{array}{c}46 \\
(333-403)\end{array}$ \\
\hline BL & $\begin{array}{c}63 \\
(299-361)\end{array}$ & $\begin{array}{c}\mathbf{1 1 1 6} \\
(341-404)\end{array}$ & $\begin{array}{c}41 \\
(257-317)\end{array}$ & $\begin{array}{c}\mathbf{4 3 4} \\
(256-315)\end{array}$ & $\begin{array}{c}25 \\
(367-435)\end{array}$ \\
\hline GA & $\begin{array}{c}45 \\
(209-266)\end{array}$ & $\begin{array}{c}86 \\
(239-299)\end{array}$ & $\begin{array}{c}\mathbf{9 6 4} \\
(177-236)\end{array}$ & $\begin{array}{c}32 \\
(179-234)\end{array}$ & $\begin{array}{c}79 \\
(257-319)\end{array}$ \\
\hline $\mathrm{ZO}$ & $\begin{array}{c}45 \\
(304-366)\end{array}$ & $\begin{array}{c}141 \\
(344-415)\end{array}$ & $\begin{array}{c}4 \\
(259-321)\end{array}$ & $\begin{array}{c}192 \\
(260-320)\end{array}$ & $\begin{array}{c}1319 \\
(378-440)\end{array}$ \\
\hline
\end{tabular}

Table 2. Contingency table of simultaneous occurrences of $Z 700$ (rows) and SLP (columns) WRs. To assess statistical confidence limits, the days were randomly shuffled 500 times. Values inside brackets span the 1 to $99 \%$ confidence level interval. Bold (italic) values correspond to statistically significant (at the $1 \%$ level) large (small) numbers of coincidences. WBL: West Blocking; other headings as in Table 1

\begin{tabular}{|lcccccc|}
\hline$Z 700 / S L P$ & AR & BL & GA & \multicolumn{2}{c|}{ WBL } & ZO \\
\hline AR & $\mathbf{9 1 2}$ & 57 & 44 & $\mathbf{4 6 3}$ & $(270-329)$ \\
& $(287-345)$ & $(323-394)$ & $(243-303)$ & $(231-291)$ & 58 & $(291-359)$ \\
BL & 28 & $\mathbf{1 0 4 1}$ & $(349-421)$ & $(263-325)$ & $(252-311)$ & 68 \\
GA & $(304-373)$ & 40 & $(248-309)$ & $(187-242)$ & $(178-234)$ & $(205-260)$ \\
ZO & 21 & 273 & 56 & 0 & $\mathbf{1 0 3 0}$ \\
& $(220-277)$ & $(354-427)$ & $(268-330)$ & $(256-317)$ & $(298-358)$ \\
\hline
\end{tabular}

Table 3. Contingency table of simultaneous occurrences of $Z 500$ (rows) and SLP (columns) WRs. To assess statistical confidence limits, the days were randomly shuffled 500 times. Values inside brackets span the 1 to $99 \%$ confidence level interval. Bold (italic) values correspond to statistically significant (at the $1 \%$ level) large (small) numbers of coincidences. Headings as in Tables 1 \& 2

\begin{tabular}{|c|c|c|c|c|c|}
\hline Z500/SLP & $\mathrm{AR}$ & BL & GA & WBL & $\mathrm{ZO}$ \\
\hline AR & $\begin{array}{c}\mathbf{6 6 3} \\
(216-272)\end{array}$ & $\begin{array}{c}64 \\
(247-305)\end{array}$ & $\begin{array}{c}62 \\
(186-238)\end{array}$ & $\begin{array}{c}\mathbf{3 5 8} \\
(176-227)\end{array}$ & $\begin{array}{c}14 \\
(200-257)\end{array}$ \\
\hline $\mathrm{BL}$ & $\begin{array}{c}37 \\
(246-306)\end{array}$ & $\begin{array}{c}\mathbf{9 2 6} \\
(276-343)\end{array}$ & $\begin{array}{c}110 \\
(211-265)\end{array}$ & $\begin{array}{c}181 \\
(200-257)\end{array}$ & $\begin{array}{c}62 \\
(233-292)\end{array}$ \\
\hline GA & $\begin{array}{c}21 \\
(192-244)\end{array}$ & $\begin{array}{c}16 \\
(222-272)\end{array}$ & $\begin{array}{c}\mathbf{7 3 1} \\
(162-214)\end{array}$ & $\begin{array}{c}\mathbf{2 2 3} \\
(153-204)\end{array}$ & $\begin{array}{c}50 \\
(180-235)\end{array}$ \\
\hline GT & $\begin{array}{c}\mathbf{2 6 1} \\
(190-237)\end{array}$ & $\begin{array}{c}242 \\
(215-271)\end{array}$ & $\begin{array}{c}85 \\
(161-211)\end{array}$ & $\begin{array}{c}\mathbf{2 6 9} \\
(154-203)\end{array}$ & $\begin{array}{c}165 \\
(174-231)\end{array}$ \\
\hline $\mathrm{ZO}$ & $\begin{array}{c}269 \\
(269-329)\end{array}$ & $\begin{array}{c}163 \\
(304-372)\end{array}$ & $\begin{array}{c}94 \\
(229-287)\end{array}$ & $\begin{array}{c}2 \\
(218-276)\end{array}$ & $\begin{array}{c}\mathbf{8 9 6} \\
(249-312)\end{array}$ \\
\hline
\end{tabular}

- Z500-SLP (Table 3)-Once again, the new (Z500) regime GT members are mainly taken from the SLP $\mathrm{AR}$ and blocking-like regimes (BL and WBL). This is in agreement with both previous comparisons, since the $Z 700 \mathrm{BL}$ regime takes members from both BL and WBL SLP regimes.

There are thus highly significant correlations between classifications of LSCs at different levels. The
GA patterns deserve some comment: they correspond to almost the same set of days at the 3 levels (the values are impressive in Tables 1 to 3 ), but the sea-level map displays an anticyclone further north, whereas there is only a ridge at higher levels, further to the south with $Z 500$ than with $Z 700$, given the high latitude of the anomaly; by contrast, the anomalies patterns (left-hand columns in Figs 2 to 5) are quite simi- 

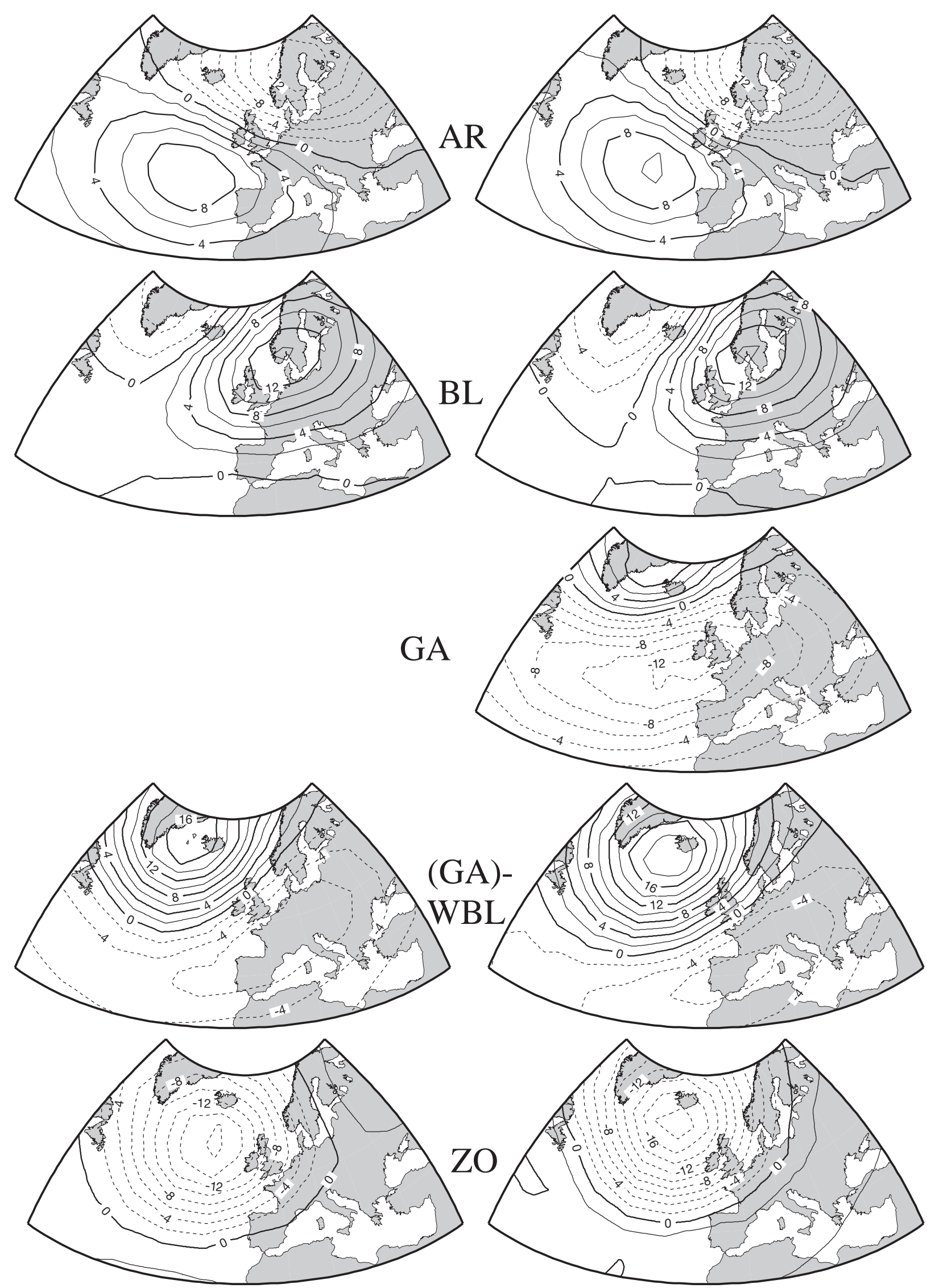

Fig. 5. The SLP WR anomaly patterns for earlier periods. Left-hand column: historical period 1880-1918. Right-hand column: 1919-1957. Labels as in Fig. 4 
lar at the 3 levels. Let us point out also that ZO days at one level are almost always classified as ZO at other levels, whereas WBL days (for SLP) never coincide with ZO days at other levels. Such conclusions were not a priori obvious since we classified all day patterns: the LSC for any given day has to belong to 1 among 4 or 5 classes, which forces classes to hold some badly correlated members. There are indeed daily patterns which do not correlate to any WR, so they display only little similarity with the central pattern of the class to which they belong. This may, for instance, explain the large percentage of BL days at all levels. This could be a major drawback; however, the conclusions of Sections 5 and 6 show that this is not the case. In any case, we take the opportunity in Section 5.4 to fix more stringent class membership conditions, with the drawback that not all days will be classified.

\subsection{SLP WRs over the last 120 yr}

In order to compare the SLP regimes for the period 1958-1997 with those obtained for the earlier periods 1880-1918 and 1919-1957, we repeated the SLP experiment over these 2 latter periods. Data were processed in the same way for each period: we first subtracted the winter mean field at each gridpoint, then performed a PCA, and finally classified the data within the space of the 10 leading PCs. The following features emerged:
-1880-1918 - The dynamical cluster algorithm selects only 4 clusters for this period. A visual inspection shows that the regime GA is no longer selected, although the WBL pattern (Fig. 5, left-hand column) has been a little distorted into a pattern somewhat intermediary between the WBL and GA patterns of Fig. 4.

- 1919-1957 - For this more recent period, 5 clusters are preferred. They are actually indistinguishable from the 5 WRs of the period 1958-1997 (compare the right-hand column anomaly maps in Fig. 5 and the left-hand column ones in Fig. 4).

This comparison of the WRs found for the last three $39 \mathrm{yr}$ periods then leads us to the important conclusion that the most recurrent LSC patterns have been very stable during at least the last $120 \mathrm{yr}$. The fact that 4 clusters are preferred for the earlier period is not a problem, since, if one nevertheless tries $k=5$ for this period, the same 5 patterns are recovered as for the final 2 periods.

Taking advantage of this stationarity, it becomes possible to classify all winter LSCs between 1880 and 1998 according to the 5 WRs of the period 1958-1997. In Fig. 6, we plot the number of occurrences of a given WR per winter, together with its 10 -yr moving average. Low frequency variability clearly appears. Very low frequency dominates in WBL and ZO. Notice, in particular, the increase of ZO (Hurrell \& van Loon 1997) over the last $25 \mathrm{yr}$ and the corresponding decrease of
GA

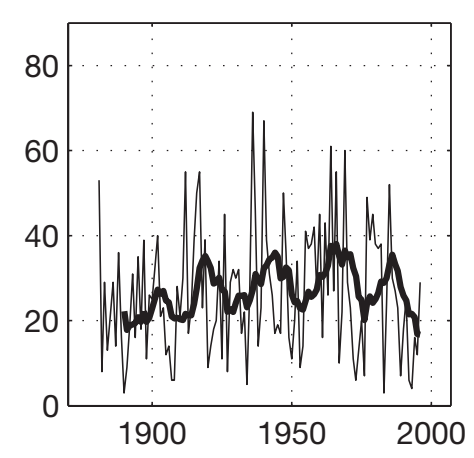

WBL

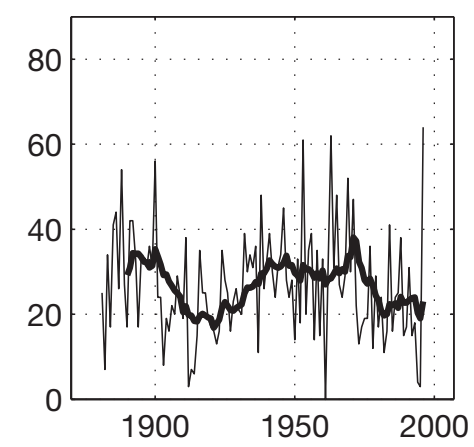

AR
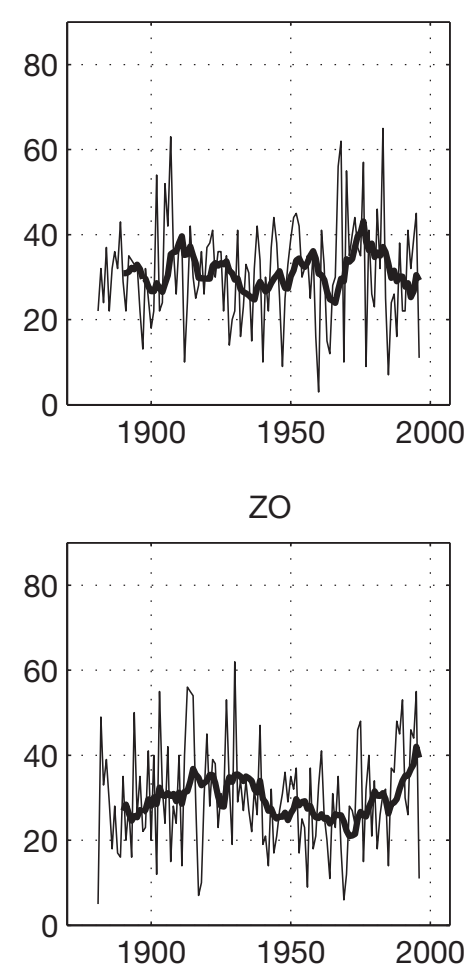

$\mathrm{BL}$

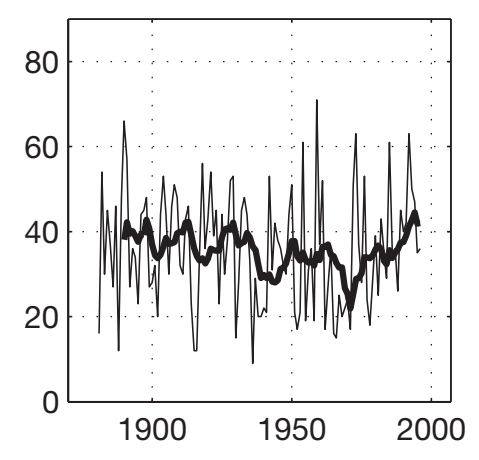

Fig. 6. Number of occurrences per winter for the 5 SLP WRs during the last $120 \mathrm{yr}$. Thin line: original signal; thick line: $10 \mathrm{yr}$ running mean. GA: Greenland Anticyclone; AR: Atlantic Ridge; BL: Blocking; WBL: West Blocking; ZO: Zonal 


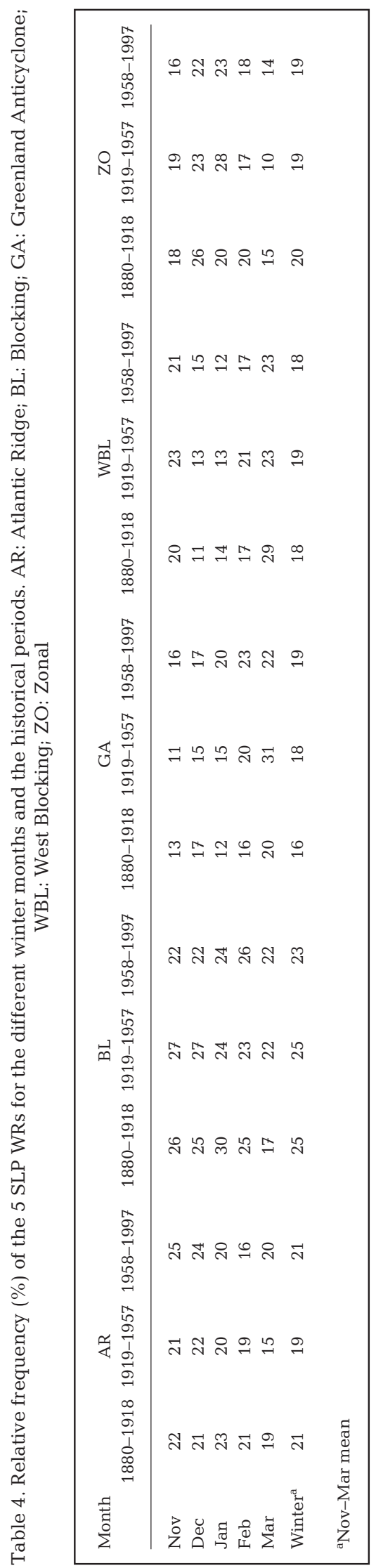

WBL, after a 'plateau' during the break in the Northern Hemisphere warming (notice, however, that it is during the recent 1995-1996 winter that one can observe the highest WBL frequency for $120 \mathrm{yr}$ !).

In Table 4, we give the average monthly frequency (in \%, from November to March) of each of the 5 SLP WRs for the 3 historical periods. The most striking features which emerge are the following: (1) When averaged over $40 \mathrm{yr}$ periods, the yearly frequencies of the WRs essentially display no significant secular trends. (2) WBL frequency displays a pronounced minimum in December and January and strongly increases during the next 2 mo. (3) GA also becomes more frequent in late winter. (4) In contrast, $\mathrm{ZO}$ frequency peaks in December and January, and then strongly decreases. One should note that all these features can be observed for the 3 periods.

\section{WEATHER REGIMES AND LOCAL TEMPERATURES}

\subsection{Methodology}

In order to determine the influence of WRs on local climate, we first classify local temperatures into terciles (warm, mean and cold). Once this has been done, we select the subset of days for which a given WR occurred and compute the local frequency of each of the previous temperature categories for this subset of days. Most often, this frequency changes and departs from its climatic value of one-third. We perform these investigations for 2 separate temperature data sets: we check the WR influence on the cold and warm tercile occurrences for both the 30 Météo-France station observed temperatures and the Western Europe NCEP reanalysed gridpoint T2m temperatures (see Section 2.2 for more details about data).

The change of frequency of a given category is computed in the following way, i.e., for the SLP WRs: once the temperatures have been classified into terciles, we compute for each gridpoint or station the $3 \times 5$ contingency table associated with the 3 temperature categories and the 5 SLP WRs: we count, for instance, the number of days with a simultaneous occurrence of each temperature category at the selected location and of the regime $\mathrm{ZO}$. We may then compute the percentage of cold days within $\mathrm{ZO}$ days and compare it to $33 \%$, the climatic percentage of cold days. If this percentage falls to say $11 \%$, we conclude that it changed by a factor $-66 \%$; notice that such changes may be larger than $+100 \%$. We also compute the $95 \%$ significance thresholds based on randomly reshuffling the days 100 times. 


\subsection{Temperatures at French stations and SLP}

The relative changes of cold day frequency for the 5 SLP WRs are displayed in Fig. 7. Non-significant departures from the climatic average correspond to the shaded areas. One of the most outstanding changes is the almost doubling ( $100 \%$ increase) of cold days with WBL at most stations (the increase is slightly lower in the south). By contrast, with ZO, cold days become very rare (the decrease is by more than $50 \%$, sometimes $75 \%$ ). Other WRs have less pronounced (but often significant) influences.

Fig. 8 shows the same changes for warm-day occurrence probability. With WBL, warm day occurrences decrease by a percentage between $50 \%$ (in the southern-most stations) and $75 \%$ (elsewhere). One can also notice the apparent paradox with AR: most stations tend with this regime to record both fewer cold and warm days; AR favours mean climatic temperatures over France!

A preliminary conclusion seems to emerge: at least for France, the WR approach allows a finer description of the local climate than mere 'average climate'; the atmosphere does not merely evolve around a mean state, it spends more times around a few specific (large-scale) states with well-defined consequences for local weather. We will see below that this conclusion also holds for most of western Europe. In the case of France, we may observe that these most frequently occurring LSC patterns induce more pronounced (either colder or warmer) departures from average climate to the north than to the south.

\subsection{Gridded European temperatures with the $Z 700$ and SLP WRs}

We display in Figs $9 \& 10$, respectively, the consequences of each $Z 700$ and SLP WR occurrence upon the cold day frequency; for brevity, we do not discuss the Z500 classification. With BL, GA and ZO, the patterns displayed in both figures are very similar, but AR gives rise to fewer cold days over most of Europe when classification is achieved with SLP. This feature may be related to the fact that the $Z 700 \mathrm{AR}$ holds many (cold) SLP WBL days, whereas the SLP AR holds a non-negligible amount of (warm) Z700 ZO days (see Table 2). GA at both levels has contrasting influences over European temperatures: the cold-day frequency increases by up to $70 \%$ over Scandinavia, obviously due to cool or cold air advection from the NW, whereas it decreases by $40 \%$ over Iberia, owing to enhanced warm air advection from the SW, due to more cyclonic conditions. Especially with SLP classification, BL is warmer from the British
Isles to Scandinavia, but much colder in south-eastern Europe; the SLP regime which we call BL is actually characterized by an anticyclonic cell over eastern Europe, providing mild oceanic flows over north-western and northern Europe, but cold air advection from easterlies over south-eastern Europe. $\mathrm{ZO}$ and WBL have their most outstanding consequences over western France, perhaps because the peculiar qualities of oceanic or continental air are most contrasting there, due to the specific LSC patterns of these WRs (Fig. 4 right-hand column). Whatever the 5 SLP WRs, the agreement between Figs 7 \& 10 (restricted to over France) is remarkable, especially when one considers the different origins and nature of the 2 data sets.

\subsection{WRs with more severe membership criteria}

Although our approach to classification implies that all the daily patterns belong to one of the clusters, we leave this requirement out for a while in order to check what happens if we prescribe more stringent class membership criteria, with the drawback that not all days will be classified. To do this, as in Robertson \& Ghil (1999), we introduce a rather severe angular filtering in the $\mathrm{PC}$ space, requiring a maximum angle of $45^{\circ}$ (apcc $>0.7$, or $\left.d_{c}<0.3\right)$ between a given day anomaly pattern and the specific central pattern of a WR. In this way, one eliminates days not belonging clearly to one of the WRs. As a consequence, no more than $28 \%$ (instead of the previous $100 \%$ ) of the patterns are classified, but this strong requirement for membership has interesting consequences. The frequencies of these 'strong' WRs range from $4 \%$ for both $\mathrm{BL}$ and WBL, up to $8 \%$ for ZO. One can see in Fig. 11 that with GA, the probability of cold days now doubles over Scandinavia; in contrast, with the reinforced ZO class, the probability of a cold day decreases by more than $80 \%$ over a large area extending from western France and south-east England northeastward to southern Scandinavia, and eastward to western Poland and Austria, including parts of the Alpine regions. WBL has similar (but opposite) strong local consequences, extending over almost all of Europe: from north-western France to Poland, the cold day probability increases by up to $130-150 \%$, when compared to its climatic average.

All these features reinforce our already stated point of view that the classification of LSCs into WRs does provide an efficient way of describing local departures of local weather from average local climate in a coherent way. WRs could even be used in downscaling approaches to local climate change investigations, starting from large-scale GCM output. 


\section{SLP (cold days)}
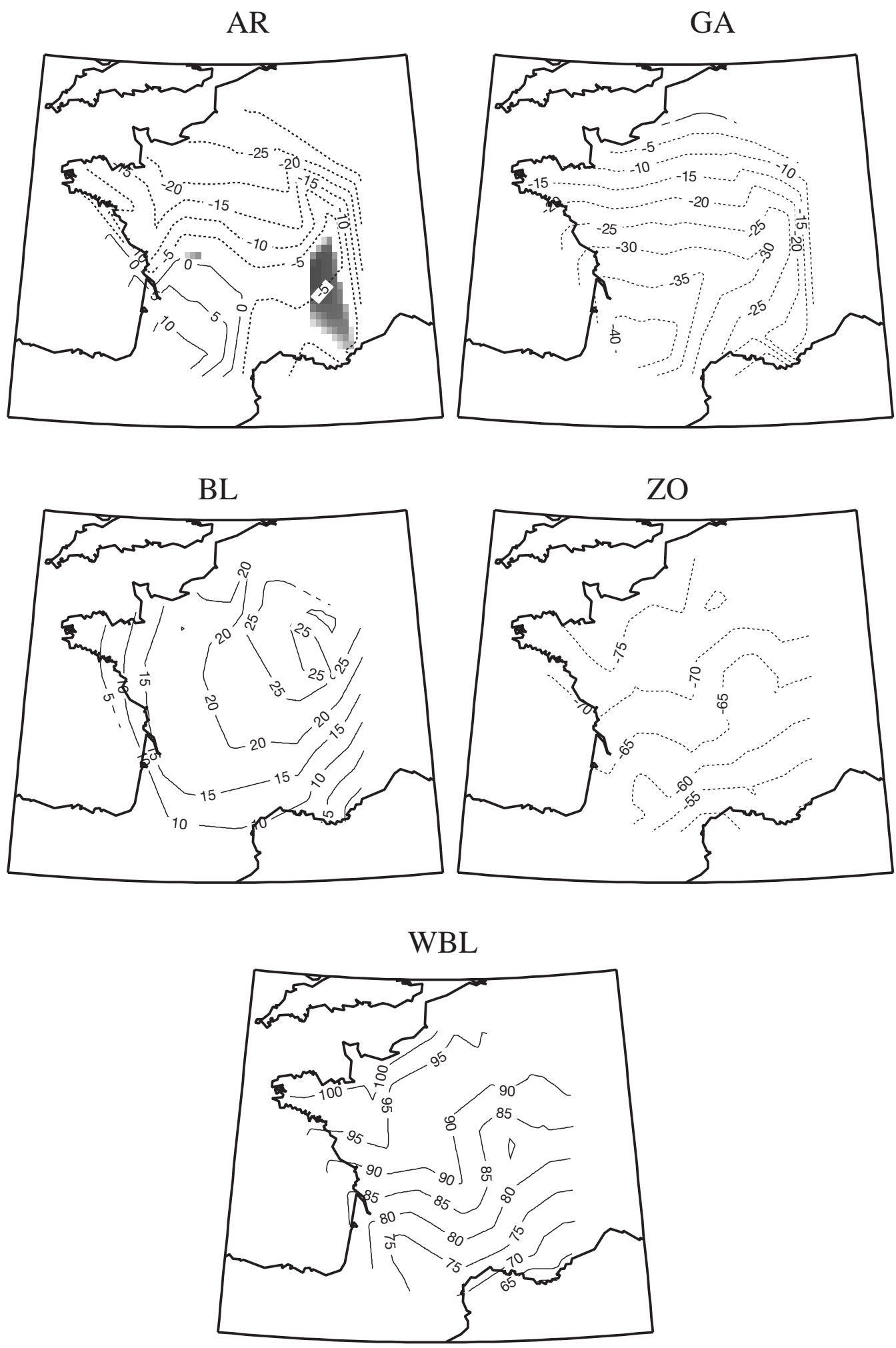

Fig. 7. Change (\%) in the frequency of cold days for each SLP WR. Solid lines: relative increase; dashed lines: relative decrease; changes are relative to the climatic mean, which is 1/3. Shaded areas: non-significant departure at the $5 \%$ level. Labels as in Fig. 4. Station data from Météo-France 


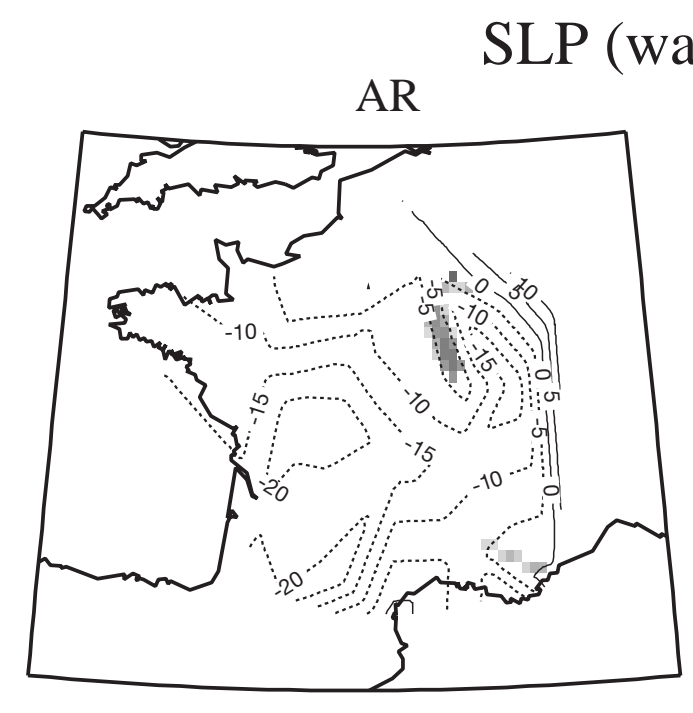

\section{SLP (warm days)}

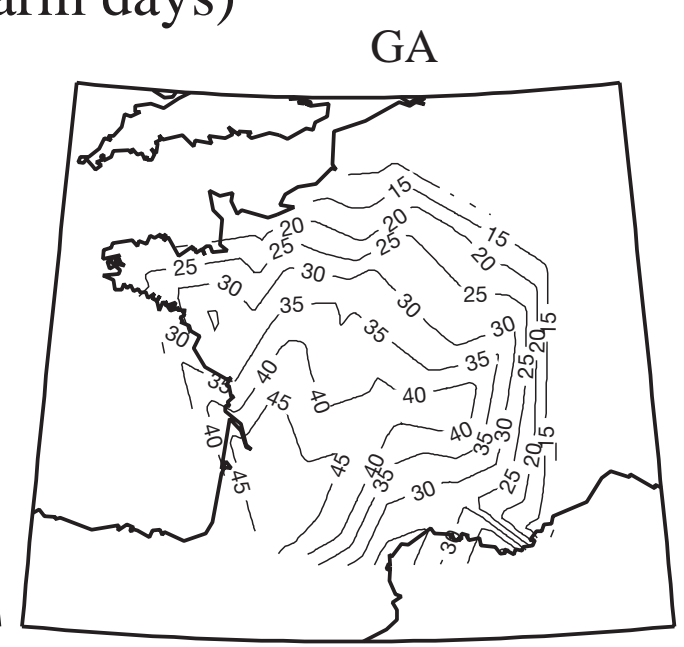

$\mathrm{BL}$
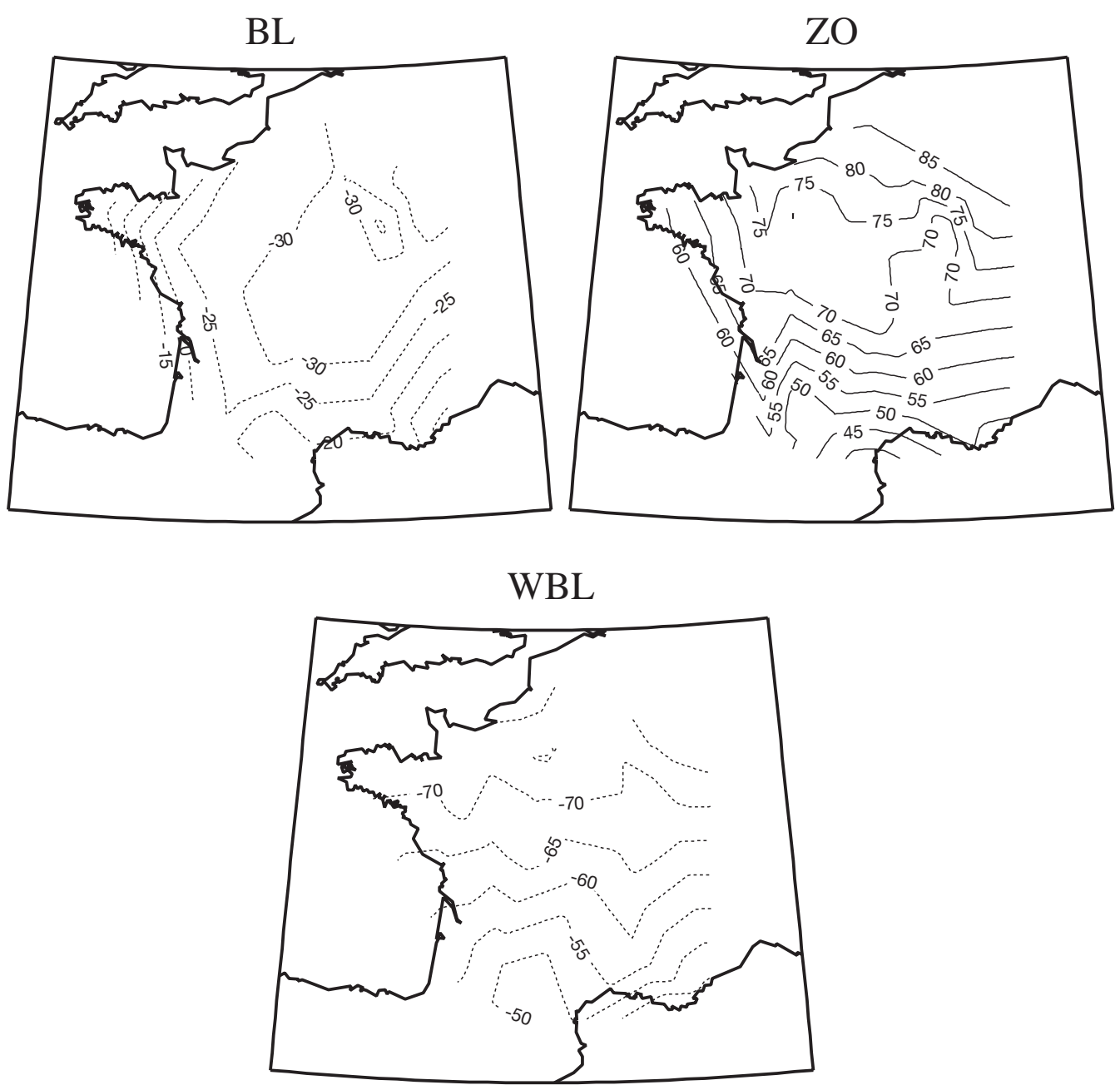

Fig. 8. Change (\%) in the frequency of warm days for each SLP WR. Solid lines: relative increase; dashed lines: relative decrease; changes are relative to the climatic mean, which is $1 / 3$. Shaded areas: non-significant departure at the $5 \%$ level. Labels as in Fig. 4. Station data from Météo-France 


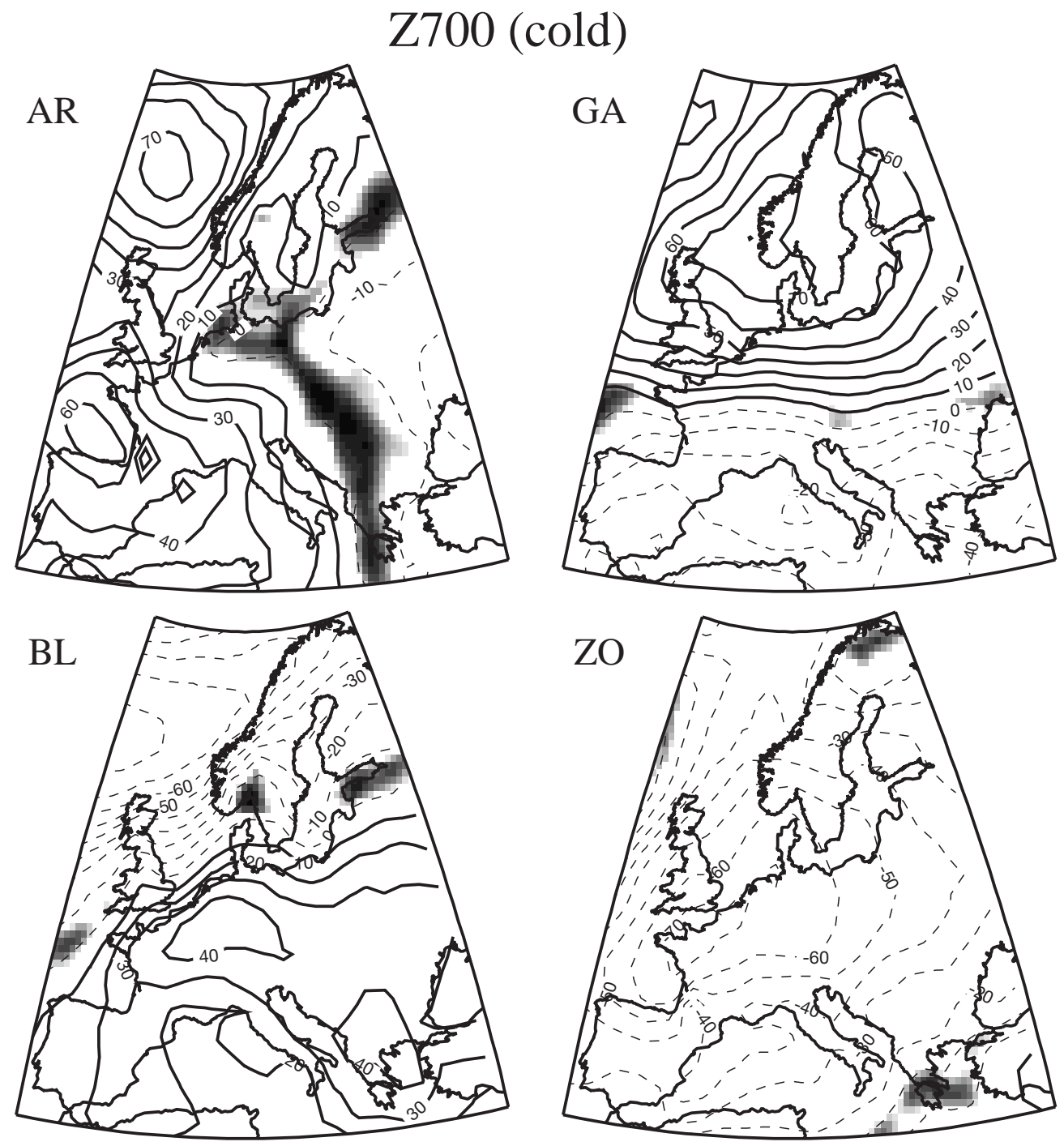

Fig. 9. Change (\%) in frequency of cold days for each Z700 WR. Solid lines: relative increase; dashed lines: relative decrease; changes are relative to the climatic mean, which is $1 / 3$. Shaded areas: non-significant departure at the $5 \%$ level. Labels as in Fig. 2. Gridded data from NCEP reanalysis

\section{Z700 WEATHER REGIMES AND LOCAL PRECIPITATION}

\subsection{Methodology}

For precipitation, 2 kinds of comparisons were performed. The first relates to the local percentage of 'wet' days (non-zero rainfall amounts), and the second to 'heavy precipitation' (hereafter HP) days, those belonging to the last decile of non-zero precipitation amounts. WR influence on wet-day frequency are investigated both for French stations and for the Alpine (APC) gridded precipitation (see Section 2.2 for details about these data). For brevity, the discussion is limited to the influence of $Z 700$ WRs.

\subsection{Wet-day percentage at French stations}

Fig. 12 shows a marked contrast between the Mediterranean region stations and all others. With AR, both western and south-eastern France experience fewer wet days, whereas the extreme north-east records up to $15 \%$ more wet days than average. This is not surprising if one considers (see Fig. 2) that during AR days, western Europe is subject to a NW flow, mostly anticyclonic to the SW, but more cyclonic to the NE. The particular orography of France may explain the small wet-day increase also observed near the Pyrenees and to the west and north of the Massif Central. With BL, the precipitation probability strongly decreases almost everywhere. The largest decrease is 


\section{SLP (cold)}
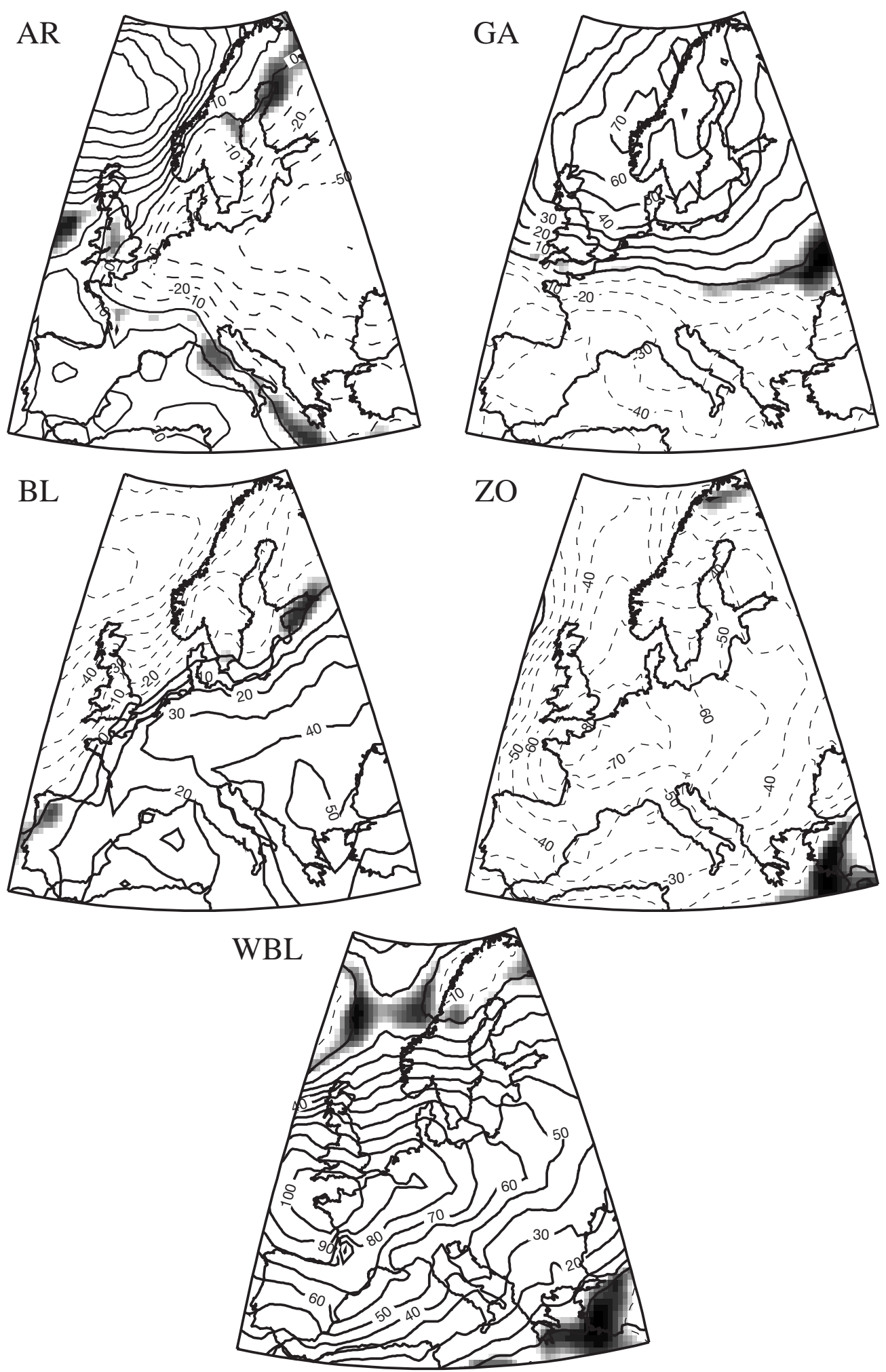

Fig. 10. Change (\%) in frequency of cold days for each SLP WR. Solid lines: relative increase; dashed lines: relative decrease; changes are relative to the climatic mean, which is $1 / 3$. Shaded areas: non-significant departure at the $5 \%$ level. Labels as in Fig. 4. Gridded data from NCEP reanalysis 


\section{SLP (cold)}
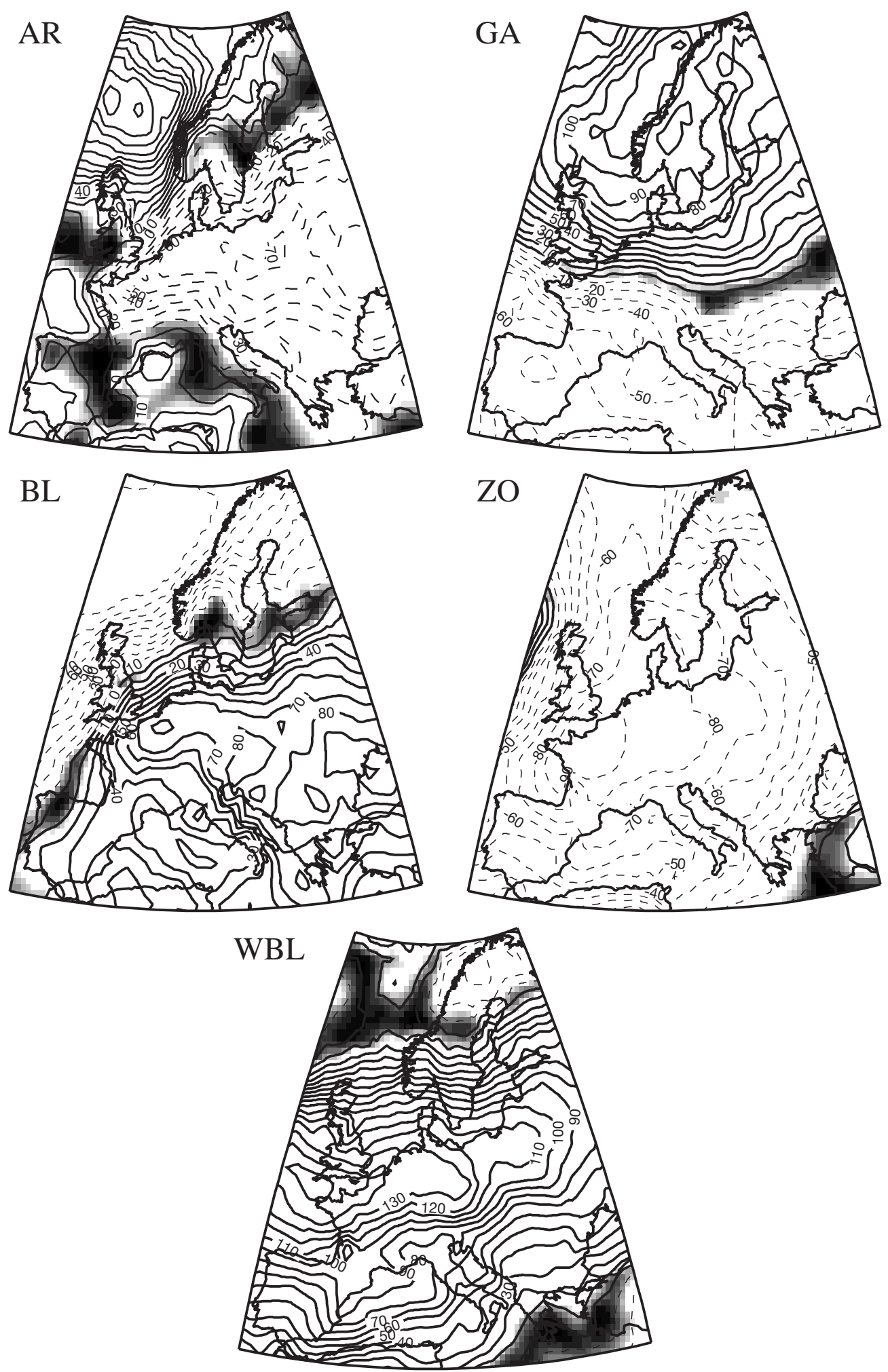

Fig. 11. Change (\%) in frequency of cold days for each $Z 700 \mathrm{WR}$, but with a more stringent cluster membership criterion through an additive angular filtering, keeping only $28 \%$ of days (see text, Section 5.4 ; compare Fig. 9). Solid lines: relative increase; dashed lines: relative decrease; changes are relative to the climatic mean, which is 1/3. Shaded areas: non-significant departure at the $5 \%$ level. Labels as in Fig. 4. Gridded data from NCEP reanalysis 

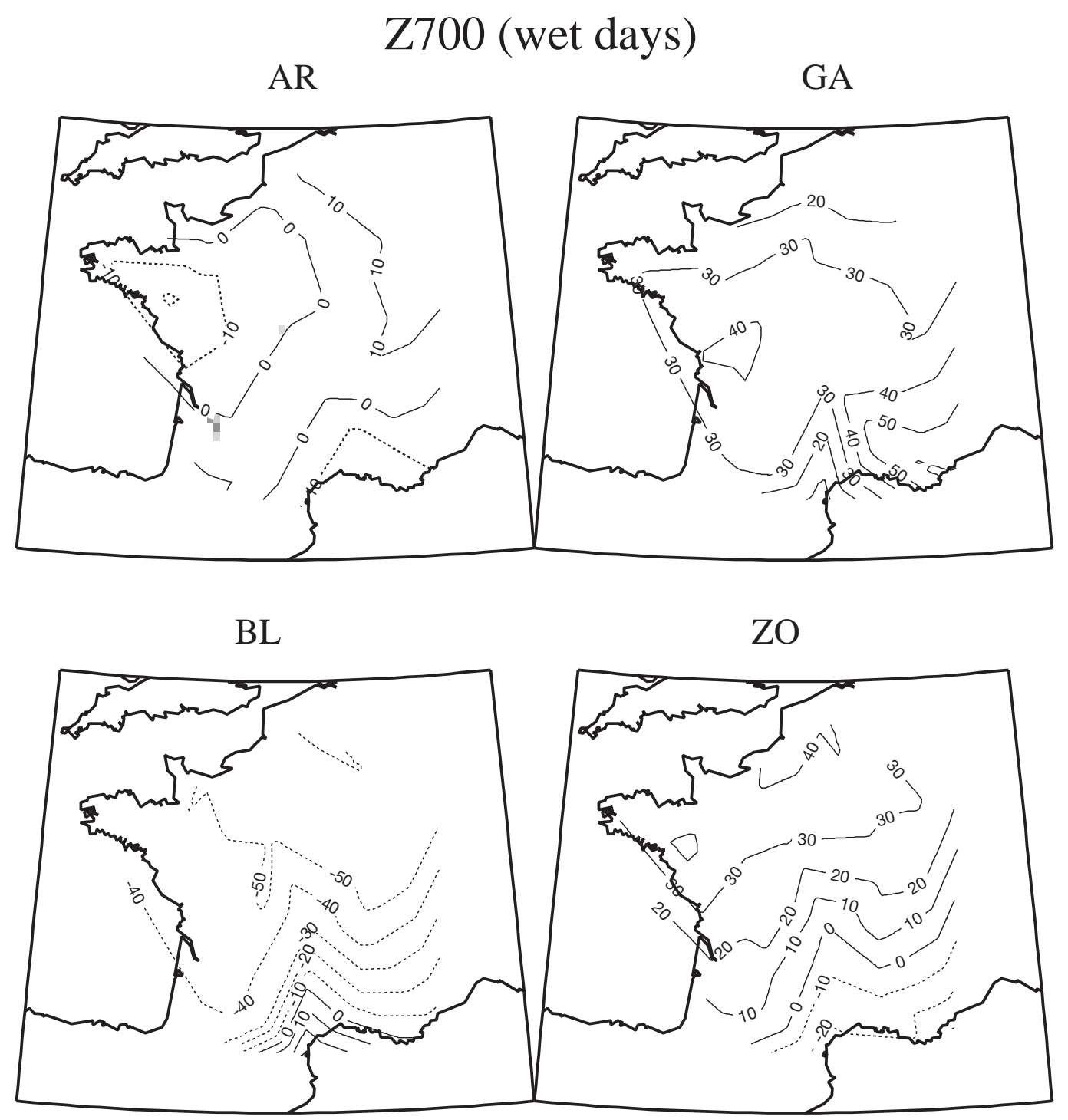

Fig. 12. Changes (\%) in wet day frequency for each $Z 700 \mathrm{WR}$, as compared to local climatic average. Solid lines: relative increase; dashed lines: relative decrease. Labels as in Fig. 2. Station data from Météo-France

greater than $60 \%$ to the north, but there is a sharp increase over the Roussillon (the French border region north of Catalonia) which is especially exposed to Mediterranean easterlies favouring intense precipitation (Plaut et al. 2001 this issue), or even extreme flooding, such as the recent ones in mid-November 1999, when precipitation amounts locally exceeded $500 \mathrm{~mm}$ in $24 \mathrm{~h}$. With GA, there is a large increase in wet days over the whole country. This increase is much smaller over the Roussillon due to lee effects, but is a maximum over the extreme south-east, where a specific class of LSC patterns which includes a low west of Brittany in addition to a Greenland high, actually favours intense precipitation (Plaut et al. 2001). Finally, with ZO days, there is a sharp contrast between the more Mediter- ranean regions and all others. Wet-day probability rises by up to $40 \%$ in the NW, but decreases by up to $20 \%$ in the SE, partially due to lee effects with the westerlies.

Correlation patterns between the November to March total precipitation and the frequency of each Z700 WR were also computed. The patterns are very similar to those of Fig. 12, which suggests again that the WR approach is relevant if one wishes to coherently describe the local climate departure from average: at least a large amount of this departure for a given period is very likely to originate in anomalous WR frequencies. We display in Fig. 13 the correlation pattern between the winter precipitation and the number of ZO occurrences. France is divided into 2 parts: one-third (roughly SE), with decreased rainfall 


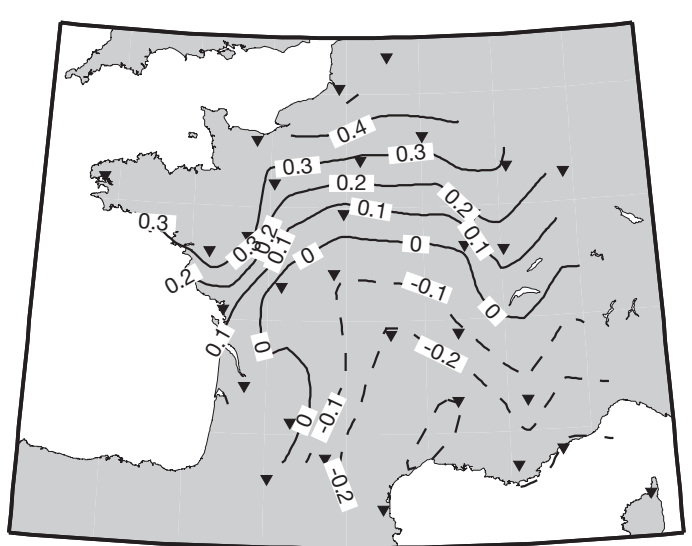

Fig. 13. Correlation between the November-March total precipitation and the frequency of the SLP ZO WR. Triangles: locations of the Météo-France stations

amounts, and the remaining two-thirds $(\mathrm{N}, \mathrm{W}$, and SW), with a positive (exceeding 0.5 at most northern stations) correlation between precipitation and the number of ZO occurrences. Such features are consis- tent with the recent increase in both the winter NAO index (Hurrell \& van Loon 1997) and the NW European countries winter precipitation (together with the corresponding decrease in precipitation in several southern Europe sub-regions [Plaut et al. 2001]).

\subsection{Wet days and heavy precipitation over the Alps}

We now investigate the influence of $Z 700$ WRs on local precipitation over the Alpine region using the APC dense gridded precipitation data. Figs $14 \& 15$ display 2 outstanding features. Firstly, many meso-structures appear, which characterize the way in which the local precipitation data are connected to LSC. Secondly, anomalies very similar to those in Fig. 12 appear for the Mediterranean regions. With BL, HP frequency decreases by roughly $80 \%$ in a wide northern third of the APC area (Fig. 15), whereas the wet-day occurrence (Fig. 14) decreases by only $30 \%$ on average. Close to Roussillon, HP probability locally increases by up to $80 \%$ : BL indeed favours intense precipitations over the

\section{Z700 (wet days)}

AR GA

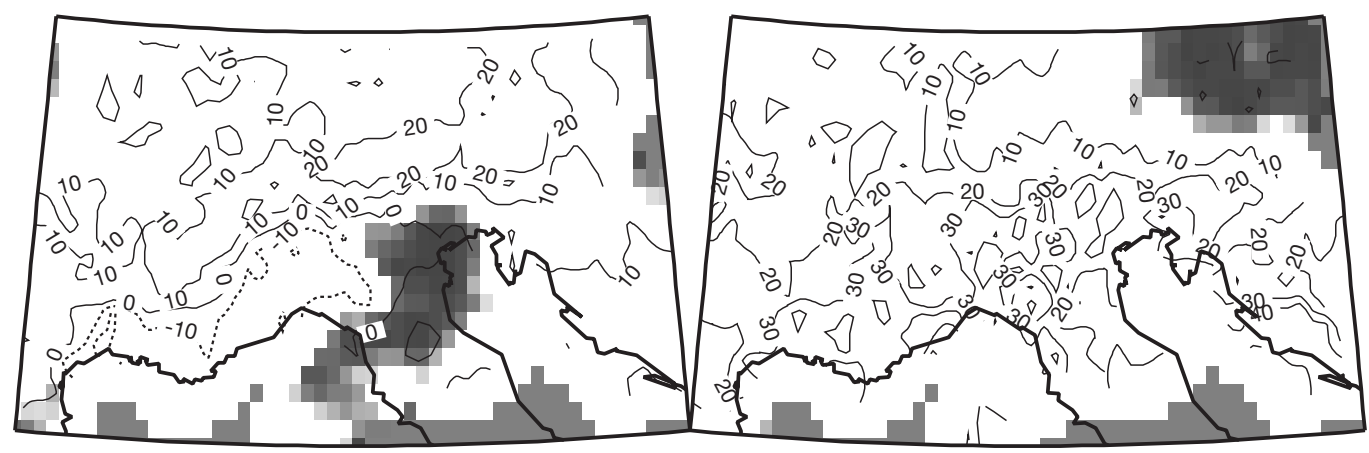

BL ZO

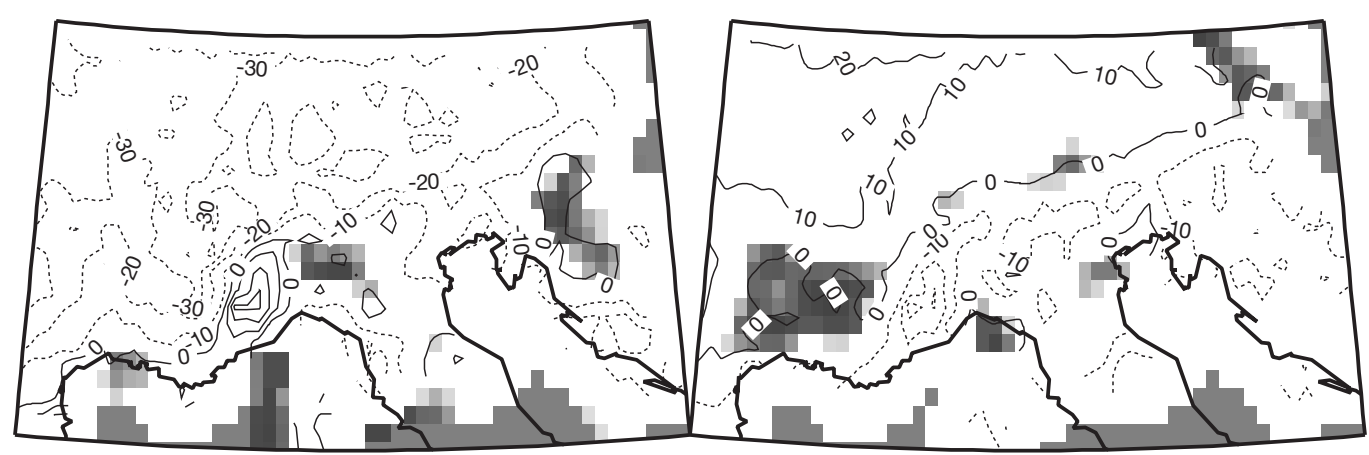

Fig. 14. Changes (\%) in wet-day frequency for each Z700 WR, as compared to local climatic average. Solid lines: relative increase; dashed lines: relative decrease. Shaded area: not significant changes at the $5 \%$ significance level. Labels as in Fig. 2. Gridded rain-gauge data from the Swiss Federal Institute of Technology ETH Zürich APC (see Section 2.2). Contour intervals: 10\% 


\section{Z700 (Heavy precip.)}

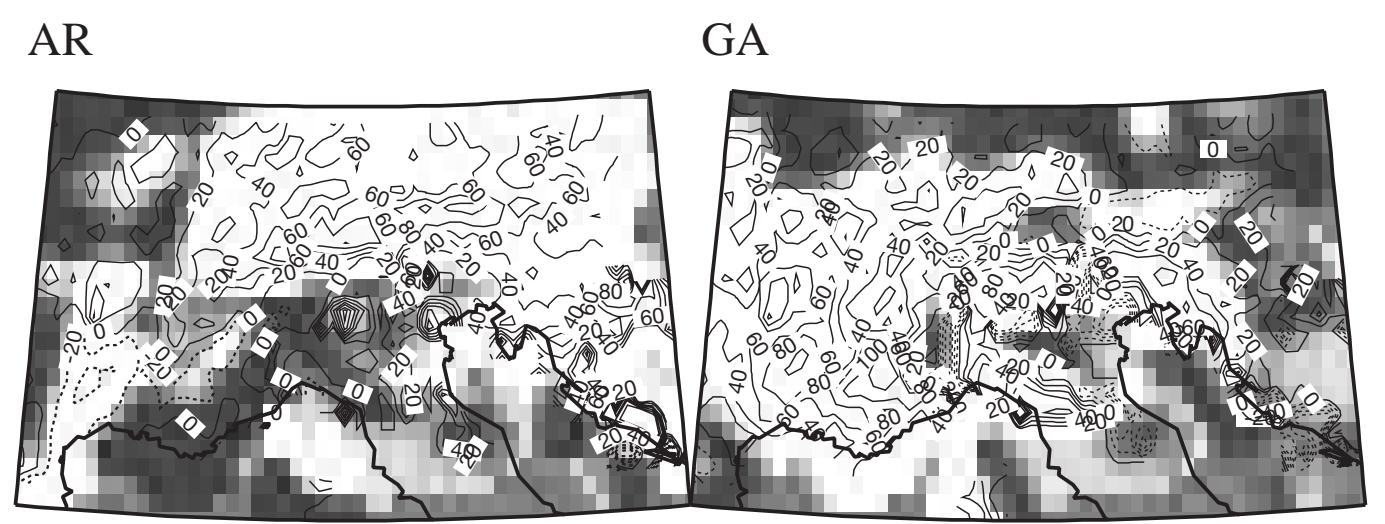

BL

$\mathrm{ZO}$

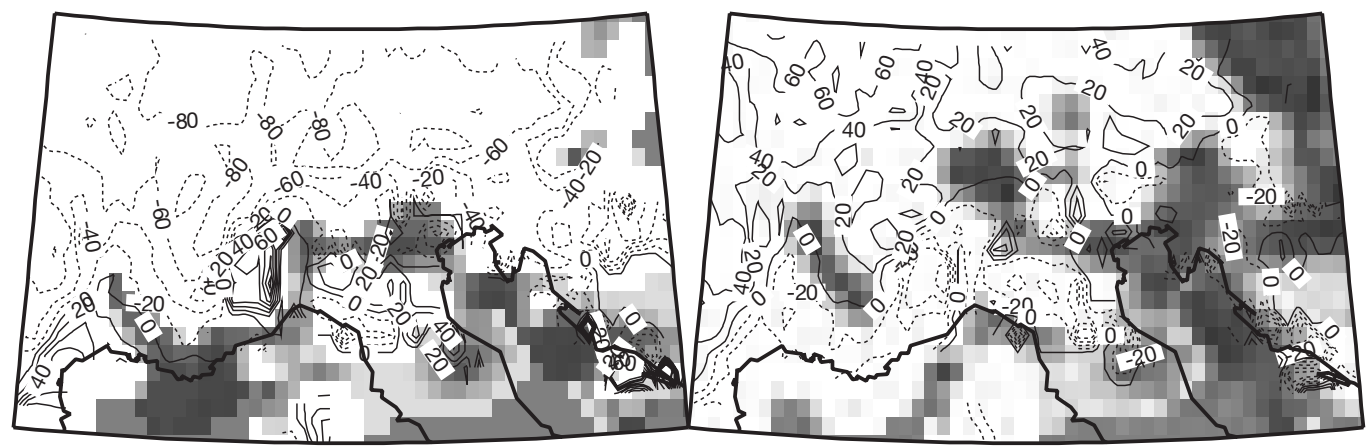

Fig. 15. Changes (\%) in frequency of heavy precipitation (last climatic decile) for each Z700 WR. Solid lines: relative increase; dashed lines: relative decrease. Shaded area: not significant changes at the $5 \%$ significance level. Labels as in Fig. 2 . Gridded rain-gauge data from the Swiss Federal Institute of Technology ETH Zürich APC (see Section 2.2). Contour intervals: $20 \%$

coastal area that is exposed to easterlies, as discussed in Section 6.2. With this WR, a large increase in both HP and wet-day occurrence probabilities is also observed over the western Italian Piedmont. For AR, HP frequency increases by almost $60 \%$ over the most northeastern third of the APC domain, where the huge amounts of snow in February 1999 was the result of persistent northwesterly flow. For GA, the increase exceeds $100 \%$ over the French southern Alps. With ZO, the HP probability increase is over $40 \%$ in a rather small north-western sector, whereas a significant decrease occurs over the French southern Alps, over Languedoc-Roussillon, but also in the Italian Piedmont.

\section{SUMMARY AND CONCLUSION}

We classified $40 \mathrm{yr}$ of winter daily Z500, Z700, and SLP LSC patterns over the North Atlantic and Europe into so-called weather regimes (WRs), using the dynamical cluster algorithm, a fully objective procedure.
Even the number of clusters was objectively established through a red noise significance test. The most significant classifications were into 4 clusters (for Z700) or 5 clusters (SLP and Z500). Classifications performed at different levels were found to be remarkably compatible. 120 yr of daily SLP maps were available. We divided them into three $40 \mathrm{yr}$ periods; each of these periods provided the same $5 \mathrm{WRs}$, although the yearly frequencies display low and very low (interdecadal) variability (Fig. 6). It was shown that the occurrence of individual WRs had specific consequences for the instantaneous departure of local weather from average local climate. In this way, a nice, objective description of local climate emerged: the atmosphere does not merely evolve around its mean state, but instead spends more time around a few characteristic recurrent states with specific consequences for local weather. Such a point of view is even strengthened by PDF calculations in the space of the 10 leading PCs where classification was performed: radial PDFs around the individual WR directions are found to be 
higher by a factor of 20 to 25 than their overall average. Whereas multimodality in planetary wave amplitude distributions (Hansen \& Sutera 1986) may be questioned (Nitsche et al. 1994), radial PDFs in our PC space display obvious maxima in the WR directions. In Section 1, we distinguished 3 categories of algorithms for the objective classification into WRs. In Section 3, we found our type 3 WRs to be almost identical to the type 2 WRs of Vautard (1990). The existence of these undeniable PDF maxima shows that they also fulfill the category 1 criterion, except for the fact that we look for them in a larger dimension space than Kimoto \& Ghil (1993a) for instance.

These conclusions and remarks reinforce the interest in dynamical-systems approaches to climate change studies, as suggested in Palmer (1993), provided the unstable fixed points may be identified unambiguously with the WRs (which seems to be the case despite minor differences). It is noteworthy that they have almost remained unchanged during the last $120 \mathrm{yr}$, whereas the time spent per year in the vicinity of each of them was possibly not stationary. The WR approach thus appears to be very likely to provide a reliable framework for building downscaling algorithms appropriate for local climate change study purposes, using GCM outputs as a proxy, since GCM-simulated LSC changes are often expected to be more relevant than mesoscale features of the same GCM.

There are many important points we could not deal with in this paper. For example, there are preferred transitions between WRs, such as ZO $\rightarrow$ AR (or BL), or WBL $\rightarrow$ BL (or GA). Transitions are extensively discussed in a companion paper (Simonnet \& Plaut 2001, this issue). One could also have combined, for example, SLP and Z700 in order to determine joint WRs. We leave this for further investigation, although we could easily check that 2-level WRs very similar to ours were obtained using simple combination rules for combining the 2 fields. Another important question may be raised: If one is no longer interested in current weather, but in intense events (if not extreme ones, for which a statistical description would be questionable), do the WRs (the most frequently observed large-scale patterns) provide an accurate framework in order to build tentative downscaling algorithms? Intense events are actually rare, so that they may be favoured by rarely occurring circulation patterns, not by the most frequent ones. We address this question in another companion paper (Plaut et al. 2001).

Acknowledgements. We thank the CRU for making their 120 yr SLP daily data set available to us, in addition to NCEP reanalyses. We also thank C. Frei and C. Schär, ETH Zürich for the Alpine Precipitation Climatology, and G.P. thanks Météo-France for the French station daily observations data set, and Lois Hoffer for carefully reading the manuscript.

\section{LITERATURE CITED}

Charney JG, DeVore JG (1979) Multiple flow equilibria in the atmosphere and blocking. J Atmos Sci 36:1205-1216

Frei C, Schär C (1998) A precipitation climatology for the Alps from high-resolution rain-gauge observations. Int J Climatol 18:873-900

Gressel W (1954a) Grosswetterlagen für den Alpenraum. Wetter und Leben, Jg 6, Heft 10-12:190-191

Gressel W (1954b) Zur Aufstellung eines synoptischen Kalendarium für den Alpenraum. Alpenraum Meteor Rundschau 7:170-194

Gressel W (1959) Zur Klassifikation der Wetterentwicklung in Alpenraum von 1946 bis 1957. Berichte des Deutschen Wetterdienstes 8 Nr54:212-215

Hansen AR, Sutera A (1986) On the probability density distribution of large-scale atmospheric wave amplitude. J Atmos Sci 43:3250-3265

Hurrell J, van Loon H (1997) Decadal variations in climate associated with the North Atlantic Oscillation. Clim Change 36:301-326

Jones PD (1987) The early twentieth century Arctic Highfact or fiction? Clim Dyn 1:63-75

Kalnay E, Kanamitsu M, Kistler R, Collins W (1996) The NCEP/NCAR 40-year Reanalysis Project. Bull Am Met Soc $77: 437-471$

Kimoto M, Ghil M (1993a) Multiple flow regimes in the Northern hemisphere winter. Part I: Methodology and hemispheric regimes. J Atmos Sci 50:2625-2643

Kimoto M, Ghil M (1993b) Multiple flow regimes in the Northern Hemisphere winter. Part II: Sectorial regimes and preferred transitions. J Atmos Sci 50:2645-2673

Lamb HH (1972) British Isles weather types and a register of a daily sequence of circulation patterns, 1861-1971. Geophys Mem 116

Michelangeli PA, Vautard R, Legras B (1995) Weather regimes: recurrence and quasi stationarity. J Atmos Sci 52: 1237-1256

Nitsche G, Wallace JM, Kooperberg C (1994) Is there evidence of multiple equilibria in planetary wave amplitude statistics? J Atmos Sci 51:314-322

Palmer T (1993) Extended-range atmospheric prediction and the Lorenz model. Bull Am Met Soc 74:49-65

Plaut G, Schuepbach E, Doctor M (2001) Heavy precipitation events over a few Alpine sub-regions and the links with large-scale circulation, 1971-1995. In: von Storch H, Jones P (eds) ACCORD: Atmospheric circulation classification and regional downscaling. CR SPECIAL 9. Clim Res 285-302

Robertson AW, Ghil M (1999) Large-scale weather regimes and local climate over the western United States. J Climate 12:1796-1813

Schepp M (1979) Witterungsklimatologie. Klimatologie der Schweiz Band III. Beiheft zu den Annalen der Schweizerischen Meteorologischen Anstalt. (Jahrgang 1978). Schweizerische Meteorologische Anstalt, Zurich

Simonnet E, Plaut G (2001) Space-time analysis of geopotential height and SLP, intraseasonal oscillations, weather regimes, and local climates over the North Atlantic and Europe. In: von Storch H, Jones P (eds) ACCORD: Atmospheric circulation classification and regional downscaling. CR SPECIAL 9. Clim Res 17:325-342

Vautard R (1990) Multiple weather regimes over the North Atlantic: analysis of precursors and successors. Mon Weather Rev 118:2056-2081 